\title{
Large-Scale Reorganization in the Somatosensory Cortex and Thalamus after Sensory Loss in Macaque Monkeys
}

\author{
Neeraj Jain, ${ }^{1,2}$ Hui-Xin Qi, ${ }^{2}$ Christine E. Collins, ${ }^{2}$ and Jon H. Kaas ${ }^{2}$ \\ ${ }^{1}$ National Brain Research Centre, Manesar, Haryana 122050, India, and 2Department of Psychology, Vanderbilt University, Nashville, Tennessee 37203
}

\begin{abstract}
Adult brains undergo large-scale plastic changes after peripheral and central injuries. Although it has been shown that both the cortical and thalamic representations can reorganize, uncertainties exist regarding the extent, nature, and time course of changes at each level. We have determined how cortical representations in the somatosensory area $3 \mathrm{~b}$ and the ventroposterior (VP) nucleus of thalamus are affected by long standing unilateral dorsal column lesions at cervical levels in macaque monkeys. In monkeys with recovery periods of 22-23 months, the intact face inputs expanded into the deafferented hand region of area $3 \mathrm{~b}$ after complete or partial lesions of the dorsal columns. The expansion of the face region could extend all the way medially into the leg and foot representations. In the same monkeys, similar expansions of the face representation take place in the VP nucleus of the thalamus, indicating that both these processing levels undergo similar reorganizations. The receptive fields of the expanded representations were similar in somatosensory cortex and thalamus. In two monkeys, we determined the extent of the brain reorganization immediately after dorsal column lesions. In these monkeys, the deafferented regions of area $3 \mathrm{~b}$ and the VP nucleus became unresponsive to the peripheral touch immediately after the lesion. No reorganization was seen in the cortex or the VP nucleus. A comparison of the extents of deafferentation across the monkeys shows that even if the dorsal column lesion is partial, preserving most of the hand representation, it is sufficient to induce an expansion of the face representation.
\end{abstract}

Key words: plasticity; spinal cord injury; dorsal column; primate; microelectrode; mapping; receptive field

\section{Introduction}

Adult brains retain a remarkable ability to change in response to injuries that interrupt transmission of peripheral inputs resulting from damage to the peripheral or central pathways (Jones, 2000; Chen et al., 2002; Jain, 2002; Kaas et al., 2008). The extent and nature of the brain reorganization depends on the extent and level of the injury. One of the earliest definitive studies on adult brain plasticity in primates showed that after transection of the median nerve to the hand in monkeys, remaining inputs to the hand expand into the deprived hand region of somatosensory area $3 b$ of cortex (Merzenich et al., 1983a,b). Since then, reorganization of the cortical maps has been demonstrated in a variety of mammalian species after different kinds of deprivations including digit or limb amputations (Merzenich et al., 1984; Wall and Cusick, 1984; Calford and Tweedale, 1988; Turnbull and Rasmusson, 1991; Florence et al., 1998), nerve transections (Wall and Kaas, 1985; Garraghty and Kaas, 1991b), dorsal root transections (Pons et al., 1991; Darian-Smith and Brown, 2000), and spinal cord injuries (Jain et al., 1997, 2000). The most extensive reorganization of the somatosensory cortex reported to date fol-

\footnotetext{
Received May 19, 2008; revised Aug. 15, 2008; accepted Sept. 18, 2008.

This work was supported by National Institutes of Health Grant NS16446 (J.H.K.) and Wellcome Trust Senior Research Fellowship 063259/Z/00/Z (N.J.). We thank Ken Catania for help during some of the recording sessions and Shashank Tandon for some of the data analysis.

Correspondence should be addressed to Neeraj Jain, National Brain Research Centre, N.H. 8, Manesar, Haryana, 122050, India. E-mail: neeraj.jain@nbrc.ac.in.

DOI:10.1523/JNEUROSCI.2334-08.2008

Copyright $\odot 2008$ Society for Neuroscience ～0270-6474/08/2811042-19\$15.00/0
}

lowed transection of the dorsal roots from C2 to T4, and after lesions of the dorsal columns at upper cervical levels. After an extensive recovery period the boundaries of the face representation shift medially into the hand region by as much as $10-14 \mathrm{~mm}$ in macaque monkeys (Pons et al., 1991) and a comparable $5 \mathrm{~mm}$ in smaller owl monkeys (Jain et al., 1997). However, the number of studies investigating extensive large-scale reorganization in primates remains few.

In both studies that examined the large-scale reorganization in the somatosensory cortex (Pons et al., 1991; Jain et al., 1997), the more medial regions of somatosensory cortex were not mapped. Thus, it is not known how far the boundary of the face representation can extend medially in area $3 \mathrm{~b}$.

The ventroposterior (VP) nucleus, the main somatosensory thalamic nucleus, also reorganizes after nerve injuries and limb amputation in monkeys and humans (Garraghty and Kaas, 1991b; Lenz et al., 1998; Dostrovsky, 1999; Florence et al., 2000). However, it is not clear if the VP nucleus is also capable of largescale reorganization as observed in area $3 \mathrm{~b}$ after dorsal column injuries.

Here, we report our results on reorganization of cortical area $3 \mathrm{~b}$ and the VP nucleus of the thalamus after unilateral lesion of the dorsal columns at cervical levels. The extent of reorganization of area $3 \mathrm{~b}$ after chronic lesions of the dorsal columns was determined in three macaque monkeys. In two of these monkeys, we mapped area $3 \mathrm{~b}$ up to the midline of the cerebral hemispheres where the foot is normally represented. In two of these monkeys, we also mapped the VP nucleus of thalamus to determine the 
Table 1. Details of the monkeys used

\begin{tabular}{|c|c|c|c|c|c|c|}
\hline Monkey & $\begin{array}{l}\text { Recovery period, exact (as rounded off in the } \\
\text { text) }\end{array}$ & Species & Sex & Age (years) & $\begin{array}{l}\text { Weight at the time of the lesion } \\
(\mathrm{kg})\end{array}$ & Lesioned side \\
\hline MCh21 & 23 months, $10 \mathrm{~d}$ ( 23 months) & M. mulatta & M & Adult, exact age not known & 8 & Right \\
\hline MCh73 & 21 months, $21 \mathrm{~d}$ ( 22 months) & M. nemestrina & $\mathrm{F}$ & $>3$ & 5.5 & Left \\
\hline MCh75 & 22 months, $14 \mathrm{~d}$ ( 22 months) & M. nemestrina & $\mathrm{F}$ & $>3$ & 4.7 & Right \\
\hline MAc19 & Acute & M. mulatta & $\mathrm{F}$ & 13 & 12 & Left \\
\hline MAc20 & Acute & M. mulatta & $\mathrm{F}$ & 4 & 5 & Left \\
\hline
\end{tabular}

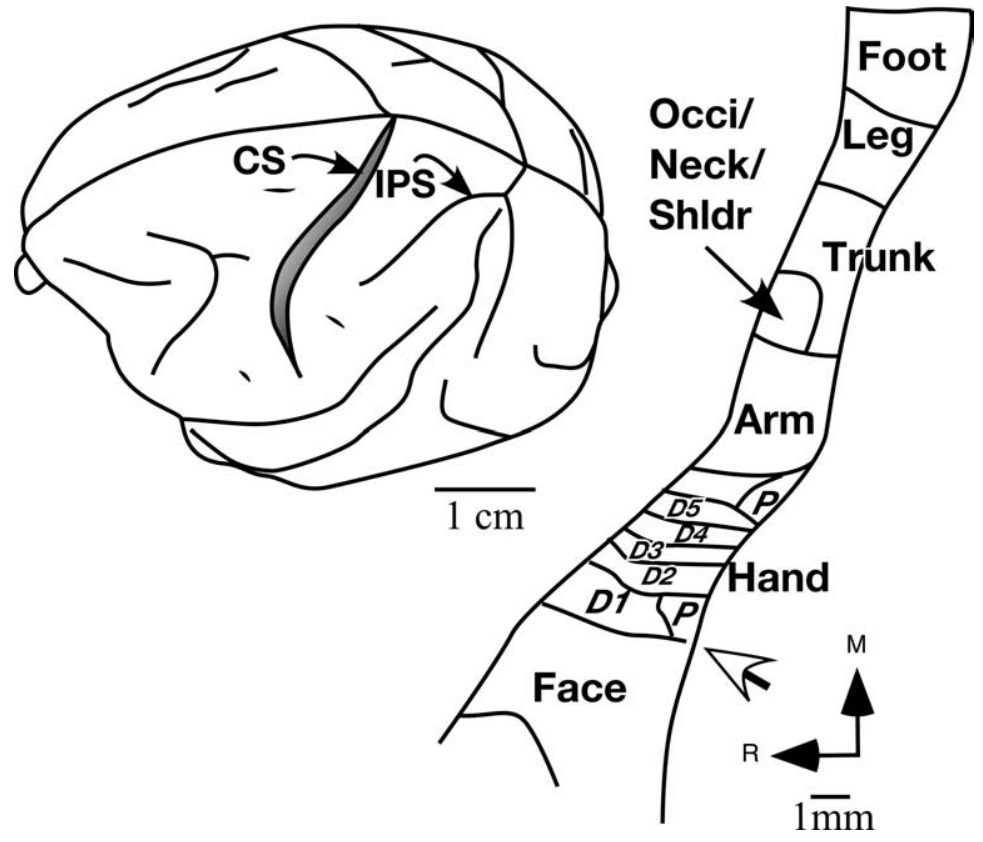

Figure 1. Location of area 3b in a macaque monkey brain (left) and outline of the somatotopy (right) in area 3b. Area 3b is located in the posterior bank of the central sulcus (CS). Inputs from the body surface are mapped in area $3 \mathrm{~b}$ in a somatotopic order with the face inputs located laterally and the hand, arm occiput/neck/shoulder, trunk leg and foot progressively medially. Part of the foot and leg region is located on the medial wall of the cerebral hemisphere (data not shown). The hand-face border (white arrow) lies at a location near the tip of the intraparietal sulcus. Some of the features of the somatotopy such as the hand-face border and the interdigital borders can be visualized histologically in the brain sections stained for myelin (Figs. 5, 11, 15). The figure shown on the left is adapted from Nelson et al. (1980). IPS, Intraparietal sulcus; Occi, occiput; Shldr, shoulder; D1, D2, etc., digit 1, digit 2, etc.; $P$, palm; $R$, rostral; $M$, medial.

extent of correspondence between cortical and thalamic reorganizations. Finally, in two additional macaque monkeys, we determined the extent of the cortical and thalamic reorganization immediately after a dorsal column lesion.

\section{Materials and Methods}

Animals. Five adult macaque monkeys were used for these experiments. Three of the monkeys were rhesus macaques (Macaca mulatta) and two were pig-tailed macaques (Macaca nemestrina). For details about each monkey, see Table 1.

Dorsal column lesions. The monkeys were anesthetized using a mixture of ketamine $(15 \mathrm{mg} / \mathrm{kg}$, i.m.) and xylazine $(0.4 \mathrm{mg} / \mathrm{kg}$, i.m. ) with supplemental doses given at one tenth of the initial dose as required. The surgical site was prepared and a midline incision was made above the dorsal spinal cord and muscles were retracted. After laminectomy the dura was incised and retracted, and dorsal columns were lesioned on one side at cervical levels using a pair of sharp fine forceps (Jain et al., 1997). The side for the lesion was selected to minimize damage to surface blood vessels. After the lesion, the dura was folded back in place and the spinal cord was covered with gel foam. The muscles and the skin were sutured in layers. The animals were given antibiotics, analgesics and dexamethasone (in reducing dose) for $5 \mathrm{~d}$ after surgery. During the recovery period the animals were carefully monitored for food and fluid intake, and any signs of self-injury. No complications were observed and recoveries were uneventful. Surgical procedure for the two monkeys with acute lesions was similar except that they were mapped immediately after the lesion. Dorsal column lesions were between C5-C8 spinal segments. Results on growth of trigeminal inputs into the cuneate nucleus from monkey MCh75 have been reported earlier (Jain et al., 2000). All experimental protocols were reviewed and approved by the Vanderbilt Institutional Animal Care and Use Committee and followed National Institutes of Health guidelines.

Microelectrode mapping. Organization of the somatosensory cortex (five monkeys) and thalamus (three monkeys) was determined by recording with microelectrodes from dense arrays of recording sites after a recovery period of 22-23 months or immediately after the lesion (Table 1). Standard multiunit mapping procedures (Nelson et al., 1980; Jain et al., 1995) were used to map the primary somatosensory cortex ( $\mathrm{S} 1$ or area $3 \mathrm{~b}$ ) and the surrounding cortex (Fig. 1) and the ventroposterior nucleus of the thalamus using parylene coated tungsten microelectrodes ( $1 \mathrm{M} \Omega$ at $1 \mathrm{kHz}$; Microprobe). For the mapping procedures, the animals were anesthetized with ketamine and xylazine as described above, supplemented with urethane $(250 \mathrm{mg} /$ $\mathrm{kg}$, i.p.). For monkey MAc19 no urethane supplement was used. The animals were given continuous infusion of IV fluids $(7 \mathrm{ml} / \mathrm{kg} / \mathrm{h})$ supplemented with dextrose $(2.5 \%)$ for $4 \mathrm{~h} \mathrm{ev-}$ ery $8 \mathrm{~h}$. The brain was exposed in the region of interest and receptive fields of neurons were determined at large numbers of recording sites. At each microelectrode recording site, the entire body was thoroughly explored for neuronal responses using cutaneous stimulation, taps and movements of joints and muscles. For area $3 \mathrm{~b}$, the electrode was inserted perpendicular to the exposed cortical surface and the receptive fields were determined at 300 $\mu \mathrm{m}$ intervals as the electrode was advanced down the cortex of the posterior bank of the central sulcus until no more responses were obtained. At many locations multiple closely spaced penetrations were made at different rostrocaudal distances from the central sulcus to explore and maximally sample the cortex of the posterior bank of the central sulcus.

For mapping the VP nucleus of the thalamus, the electrode was inserted in the stereotaxically vertical plane and advanced manually to the dorsal surface of the thalamus. Thereafter the electrode was advanced using a hydraulic microdrive (Kopf) and the receptive fields of neurons were determined every 200 or $300 \mu \mathrm{m}$. Each of the sites where receptive fields were determined in the cortex or the thalamus is called a recording site. The total number of penetrations made in each monkey was based on our goal to sample the desired extent of area $3 \mathrm{~b}$, and the VP nucleus, while maintaining an optimal experimental duration. At the end of the mapping procedures, electrolytic lesions were made at selected locations to aid the identification of electrode penetration sites in sections of the cortex (Fig. $2 B$ ) or thalamus (Fig. 3). To determine the trajectory of the electrodes, con- 
tinuous lesions were made in a few penetrations by continuously passing a $10 \mu \mathrm{A}$ current as the electrode was withdrawn at the rate of $25 \mu \mathrm{m} / \mathrm{s}$ (Fig. 2B).

To identify the path of the electrode tracks in the cortex of monkeys MCh73 and MCh75 where the cortex was flattened (see below), we made electrolytic lesions and we coated the electrode with DiI $\left(1,1^{\prime}\right.$, di-octadecyl-3,3,3' $3^{\prime}$ tetramethylindocarbocyanine perchlorate) or DiO (3,3'-dioladecyloxacarbocyanine perchlorate) before some of the penetrations toward the end of the experiment. The sections of the flattened cortex (see below) were temporarily mounted on glass slides in glycerol and examined under fluorescent microscope equipped with a drawing tube to draw the electrode tracks labeled with DiI or DiO (Fig. 2A). Blood vessel pattern and other tissue artifacts were also drawn using a dark field microscope for later matching the electrode tracks with the myelin-stained sections. For other details of the mapping procedures and reconstruction of the maps, see Nelson et al. (1980), Kaas et al. (1984), and Jain et al. (1995).

Histology. The monkeys were perfused transcardially with buffered, $\mathrm{pH} 7.5,2$ or $3 \%$ paraformaldehyde, followed by buffered paraformaldehyde with $10 \%$ sucrose and finally with $10 \%$ buffered sucrose. The brain was removed, and the cortex was separated from the underlying tissue, blocked, and cryoprotected in 30\% sucrose. For the monkeys MCh73, MCh75, and MAc19, the somatosensory cortex was flattened between glass slides after carefully opening the sulci. The flattened cortex was frozen and cut parallel to the pial surface on a sliding microtome into $40 \mu \mathrm{m}$ thick sections. The sections were stained for myelin to reconstruct electrode penetrations sites and to define the anatomical boundaries of the hand and face representations (Jain et al., 1998, 2001). For the remaining two monkeys MCh20 and MAc20 the cortex was cut into $50 \mu \mathrm{m}$ thick sections in a plane perpendicular to the central sulcus, which is slightly off the parasagittal plane. Different series of sections from the cortex were stained for Nissl substance, myelin (Jain et al., 1998), and cytochrome oxidase activity (Wong-Riley, 1979). The spinal cords of the three monkeys with chronic lesions were cut in a parasagittal plane at a thickness of 40 or $50 \mu \mathrm{m}$ for reconstruction of the lesion. For the two acute cases, the spinal cord was cut in a transverse plane into 50 - or $60-\mu \mathrm{m}$-thick sections.

Reconstruction of the lesion site. Drawings of the spinal cord sections in the region of the lesion were made using a microscope equipped with a camera lucida, or the images of the sections were acquired using a digital camera attached to a microscope. The maximal extent of the lesion, the white matter and the gray matter were measured and the spinal cord was reconstructed in the coronal plane. For the acute cases all the transverse spinal cord sections with lesion were drawn and overlaid on a transparency sheet to define the maximum extent of the lesion.

\section{Results}

We first present results on the reorganization of area $3 \mathrm{~b}$ of the monkeys with chronic and acute lesions, and then on the reorganization of the ventroposterior nucleus of the thalamus.
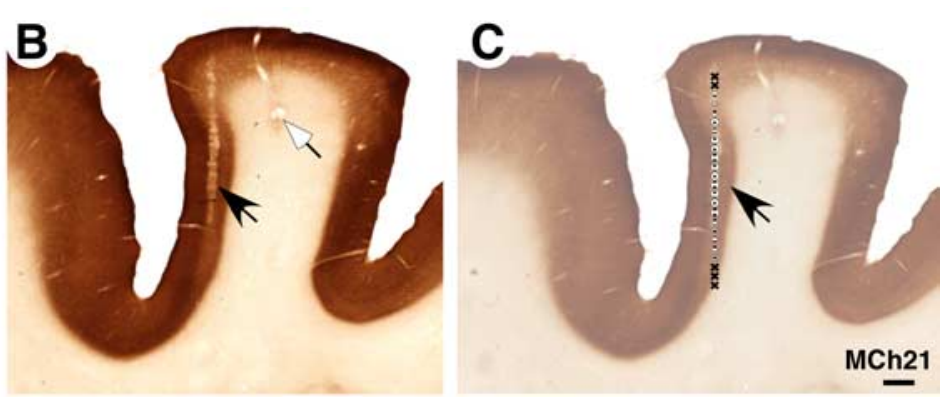
(see Materials and Methods) marking the electrode track (black arrow) in area 3b of the posterior bank of the central sulcus (white arrow). The section of the cortex was cut in a plane perpendicular to the central sulcus and stained for cytochrome oxidase. $\boldsymbol{C}$, An example of recording sites along the electrode tract of the section shown in $\boldsymbol{B}$ (black
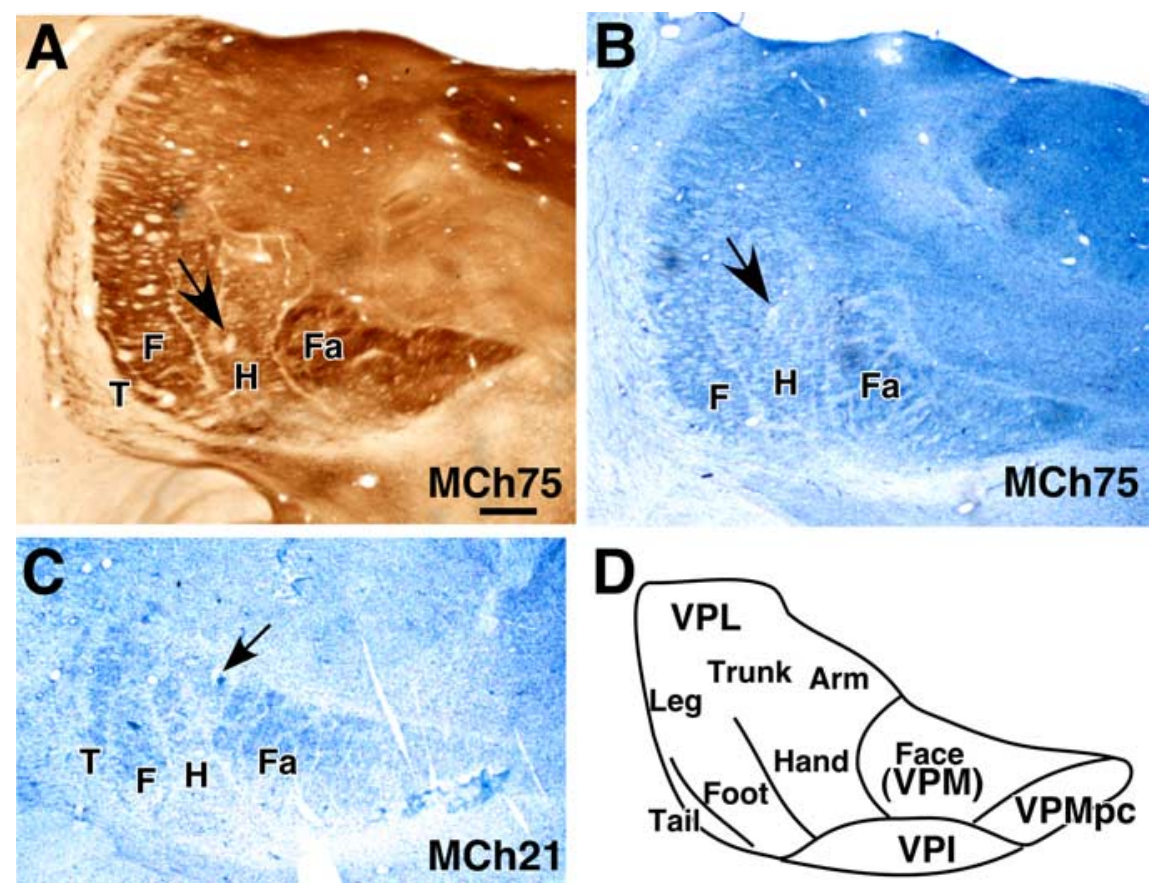

Figure 3. $\boldsymbol{A}-\boldsymbol{C}$, Coronal sections of the thalamus through the VP nucleus of monkey MCh75 $(\boldsymbol{A}, \boldsymbol{B})$ and $M C h 21(\boldsymbol{C})$ showing electrolytic lesions (arrows) made to help align microelectrode recording results with histological features revealed in sections Note the lines separating the representations of major body parts can be seen as $C 0$ light septa in $\boldsymbol{A}$ and cell sparse septa in $\boldsymbol{B}$ and C. Fa, Face; H, hand; F, foot; T, tail; VPMpc, parvicellular subdivision of the VP nucleus; VPI, ventroposterior inferior nucleus. The tail subnucleus is not apparent in the section shown in $\boldsymbol{B}$. Scale bar (in $\boldsymbol{A}$ for $\boldsymbol{A}, \boldsymbol{B}, \boldsymbol{C}$ ), $1 \mathrm{~mm}$.

\section{Reorganization in area $3 b$}

In macaque monkeys, area $3 \mathrm{~b}$ forms a mediolateral strip of cortex along in the posterior bank of the central sulcus (Fig. 1). In area 3b, there is an orderly representation of somatosensory inputs from the contralateral body surface with the foot medial most, followed laterally by representations of the leg, trunk, arm and the hand (Nelson et al., 1980). Medially, parts of the foot and leg representations extend onto the medial wall of the cerebral hemisphere. Lateral to the hand representation, starting at a location near the end of the intraparietal sulcus, trigeminal inputs from the face followed by those from the oral cavity are represented. The border between the hand and the face representations, as well as borders between the digit representations, can be visualized in histological sections of the flattened cortex stained for myelin (Jain et al., 1998, 2001; Qi and Kaas, 2004). 


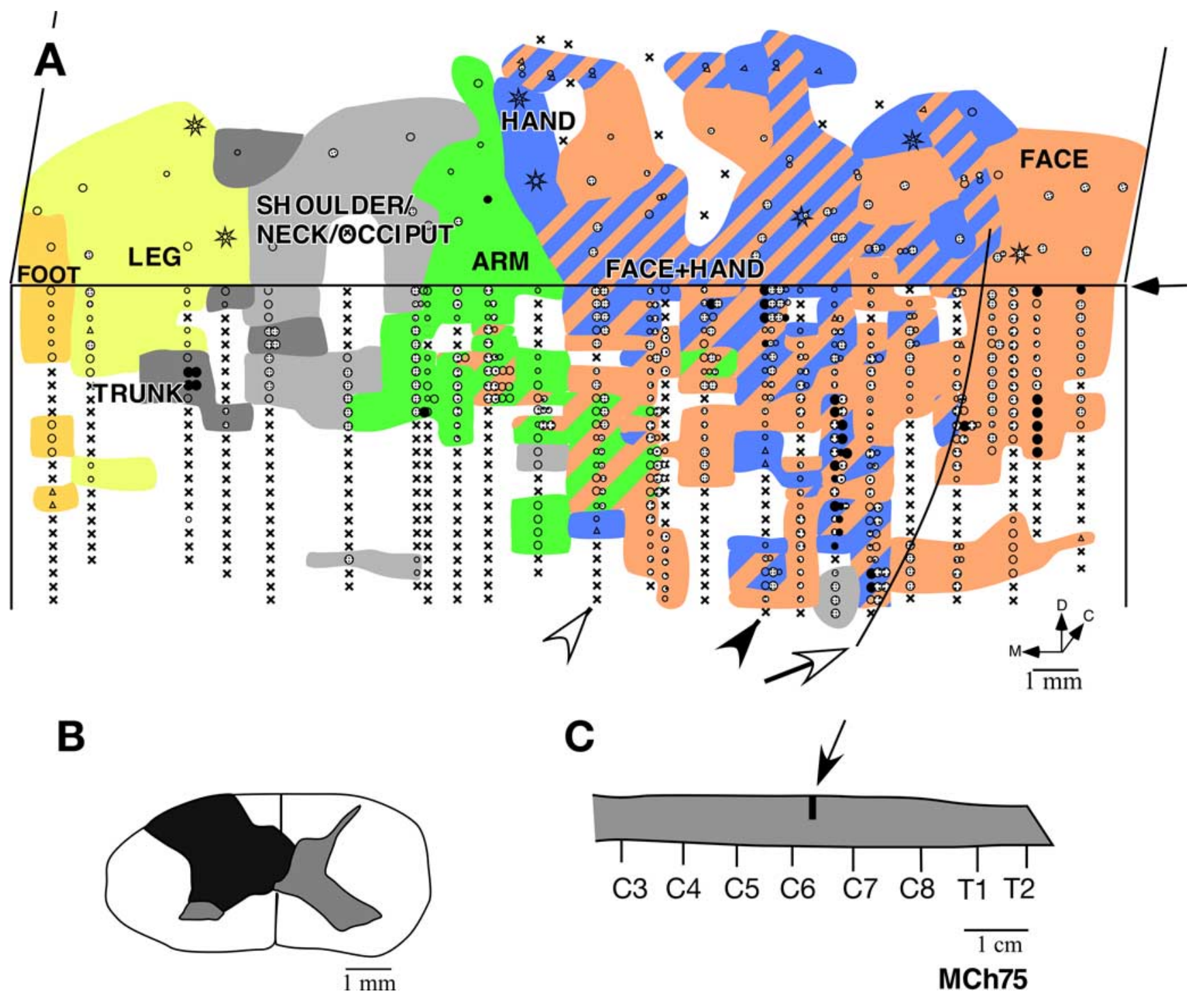

Figure 4. A, Somatotopy in left area $3 \mathrm{~b}$ and the adjacent area 1 in monkey MCh75. The border marked by the white arrow is the normal hand-face border revealed in sections of the flattened cortex stained for myelin (Fig. 5). Note the expansion of the face and the arm responsive regions into the deafferented hand area. The black arrow on the right marks the line drawn at $1000 \mu \mathrm{m}$ from the surface. The recording sites below the line are on the posterior bank of the central sulcus, and the sites above the line are on the dorsal surface of the postcentral gyrus. Regions devoted to different body surfaces are color-coded. In the striped regions, neurons responded to two or more body parts as per the color codes. The arrowheads mark the position of penetrations for which the receptive fields are shown in Figure 7. Black circles indicate sites with response to light cutaneous touch on the skin, dotted circles with responses to hairs, open circles with responses to taps. Larger circles indicate better responses and smaller circles weaker responses. Triangles mark the sites with weak responses to hard taps, making it difficult to define the receptive field with certainty. Multiple dots indicate sites with multiple or split receptive fields. The crosses mark the sites where neurons did not respond to any stimulation. Stars mark the sites where electrolytic lesions were made. D, Dorsal; $M$, medial; C, caudal. $B$, Reconstruction of the spinal cord lesion (black) on a coronal section. There was some sparing in the medio-dorsal region corresponding to the location of the fasciculus gracilis. C, Location of the lesion (arrow) shown on the outline diagram of the spinal cord. Rostral is to the left. C3, etc., denote the cervical roots and T1 is the first thoracic root.

Reorganization of area 36 in monkeys with recovery periods of 22-23 months

In area $3 \mathrm{~b}$ of the three monkeys with long recovery periods we observed a medial expansion of the region responsive to tactile stimulation of the contralateral face. The extent of cortical reorganization and the nature of the recorded receptive fields varied for each monkey depending on the extent and level of the lesion as described below.

Monkey MCh75. This monkey, which had the lesion at the level of C6/C7 (Fig. 4C), was mapped 22 months after the spinal cord lesion. A lesion at this level is expected to remove most of the inputs from digits 3,4 , and 5 , while preserving those from most of the palm, digits 1 and 2 and the anterior arm (Sherrington, 1939; Florence et al., 1988; Flor et al., 1997; Darian-Smith and Ciferri, 2005). The lesion was partial (Fig. 4B). There was sparing of part of the dorsal columns on the medial side at the location of the fasciculus gracilis. The sparing of much of fasciculus gracilis preserved inputs from the lower body. The spinal cord damage extended to portions of the ipsilateral dorsal and ventral horns, but did not damage other fiber tracts.

Results from mapping of area $3 \mathrm{~b}$ show that the face responsive region extended into the hand cortex and somewhat beyond into the region of the arm representation (Fig. $4 A$ ). The expansion was $\sim 12$ $\mathrm{mm}$ medial to the normal hand-face border. We ascertained the normal border between the hand and the face representations (Fig. $4 \mathrm{~A}$, open arrow) by staining sections of the flattened cortex for myelin, which clearly shows this border as a myelin-light septum (Fig. 5). We have previously shown that this border remains immutable despite cortical reorganization after deafferentations (Jain et al., 1998). 


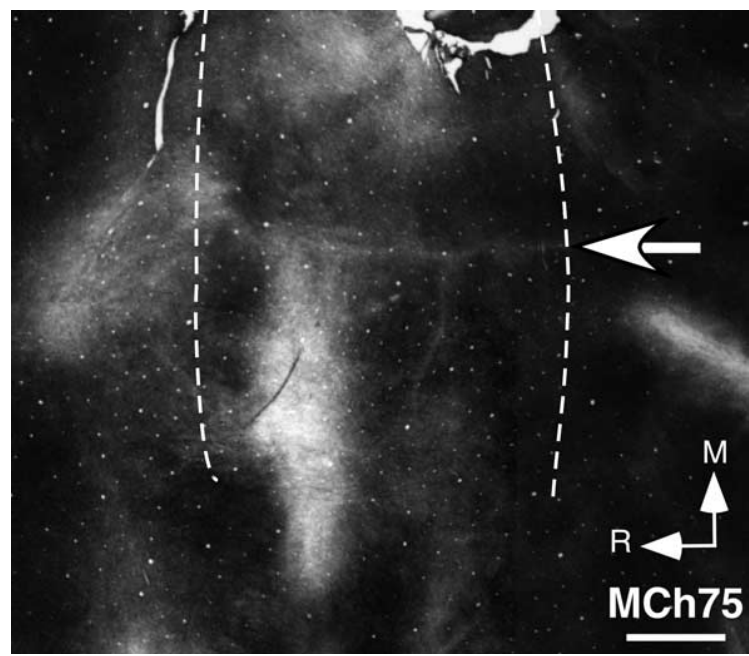

Figure 5. Photomicrograph of a section of the flattened cortex of monkey MCh75 stained for myelin. The section was cut parallel to the pial surface. The myelin-light septum (arrow) marking the hand-face border is clearly visible. Left and right dotted lines respectively mark the fundus and the lip of the posterior bank of the central sulcus. R, Rostral; M, medial. Scale bar, 2 $\mathrm{mm}$.
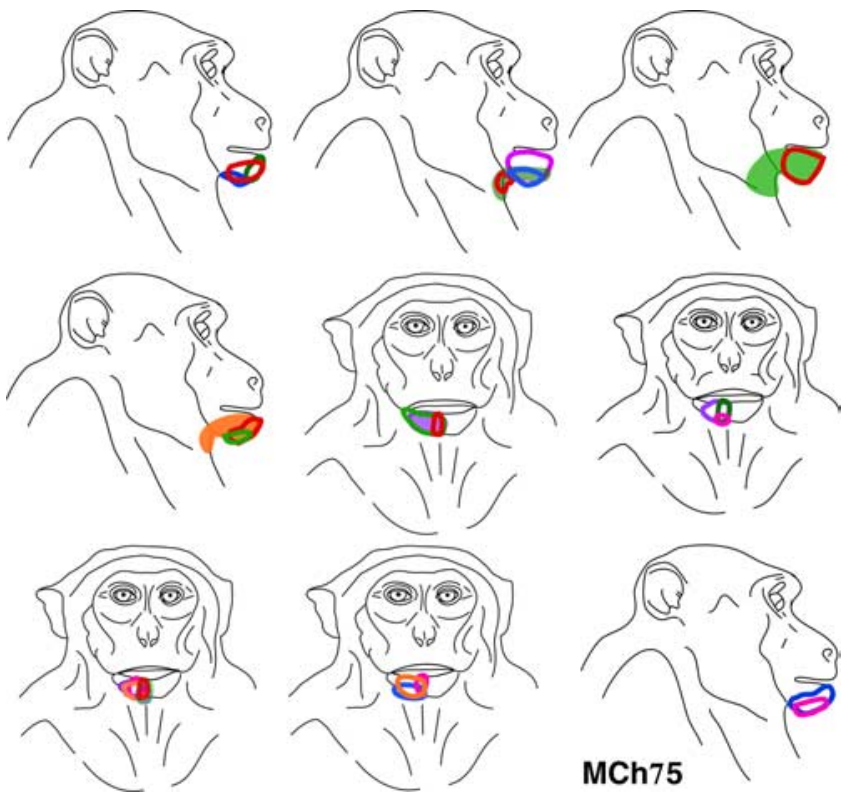

Figure 6. Locations of all the receptive fields on the face that were recorded for neurons in the reorganized cortex medial to the normal hand-face border in monkey MCh75 (Fig. 4A). Note that all the receptive fields were confined to the chin except for a few that extended to the posterior jaw and neck.

Within the hand region, at a majority of the mapping sites the neurons responded to either the stimulation of the face alone, or to the stimulation of both the face and the hand. Of 105 recording sites where neurons responded to touch on the hand, at only 16 (i.e., 15.2\%) of the sites the receptive field was restricted to the hand. At the remaining 89 sites the receptive fields included skin on both the hand and the face. All the receptive fields on the face in the expanded face representation, i.e., medial to the normal hand-face border, were on the chin (Fig. 6). A few of these receptive fields also extended to the neck area. The neurons usually responded to vigorous stimulation of the hairs on the chin or to light taps. In the normal face region, lateral to the hand-face
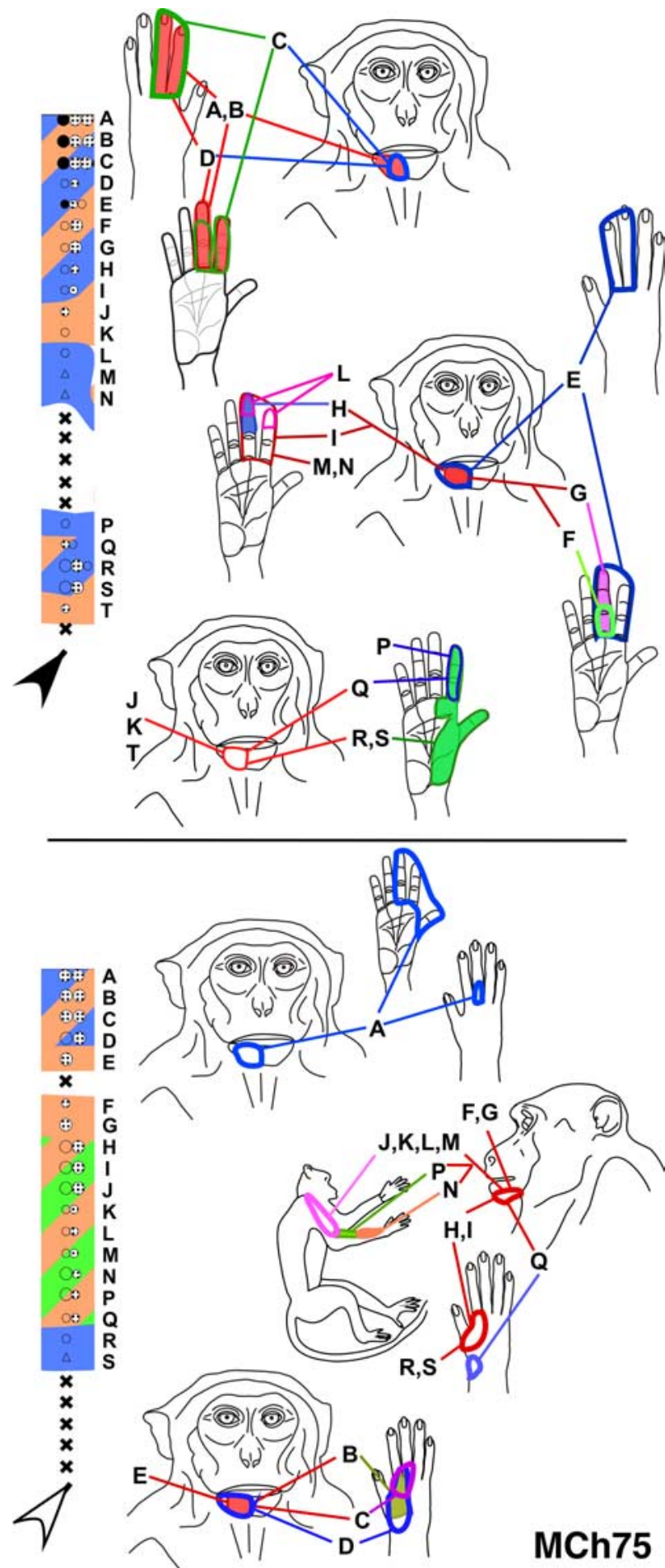

Figure 7. Receptive fields of neurons in two penetrations through the posterior bank of the central sulcus in monkey MCh75. The penetrations illustrated here correspond to those marked by the black and the open arrowheads in Figure 4 and use the same color codes. The receptive fields of neurons at the sites marked by letters are shown on the figurines on the left. Note that at many sites the receptive fields split with responses elicited by touch on the hand/arm as well as chin. The receptive fields on the hand are large, often extending over multiple digits, unlike those in normal animals. For other conventions, see legend to Figure $4 \mathrm{~A}$.

border, we found neurons with receptive fields distributed all over the face as expected (data not shown).

The receptive fields on the hand were confined to the radial half: D1, D2, D3, and the radial palm (Figs. 7, 8). The sparing of 


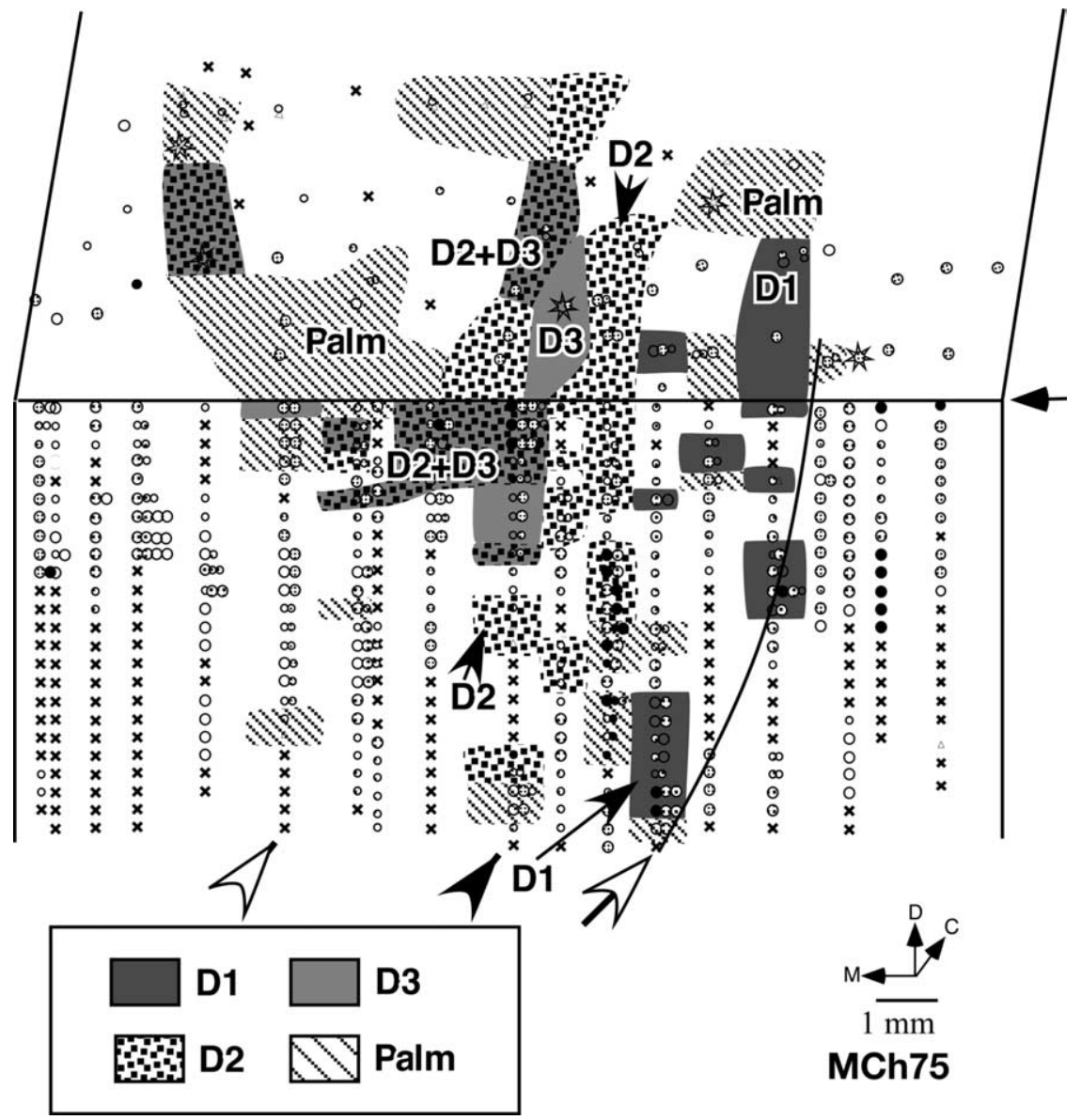

Figure 8. The organization of the hand representation in monkey MCh75. No clear somatotopy with distinct borders between digits was found although a lateral to medial D1-D3 trend was present. The white arrow marks the position of the septum that demarcates the hand-face border (Fig. 5). The arrowheads mark the penetrations for which detailed receptive fields are shown in Figure 7. Other conventions are as for Figure $4 A$.

the radial hand inputs corresponds to the level of the lesion as these inputs enter the spinal cord rostral to C7 (Sherrington, 1939; Florence et al., 1988, 1989). However, the somatotopy of the spared hand inputs was highly abnormal. The receptive fields were large, often encompassing one or more complete digits, and even parts of the palm (Fig. 7). The neuronal responses were usually weak and required light taps on the hand. In contrast, the receptive fields in the hand region of area $3 \mathrm{~b}$ of normal macaque monkeys are generally very small, and are confined to a small portion of the skin of a phalanx of a digit, requiring only light cutaneous touch for neuronal activation (Nelson et al., 1980; Pons et al., 1987). Although there is an overall latero-medial trend of topography in the hand region from D1 to D3, the pattern of discrete representations of the digits was completely disrupted (Fig. 8), indicating that a reorganization within the hand region occurred as a result of the partial deafferentation of hand inputs.

In this monkey, we also found many recording sites where neurons responded to the stimulation of both the arm and the face. There were a total of 59 sites with cells responsive to stimulation of the arm. Out of these, neurons at 23 sites had responses to stimulation of the chin as well as the arm. The receptive fields on the arm were found on the anterior upper arm and radial forearm for which inputs enter the spinal cord rostral to the level of the lesion at C6/C7 (Sherrington, 1939; Dykes and Terzis, 1981). Moreover, the arm region did not form a sharp boundary with the hand region. There was considerable interdigitation of the hand and arm responsive areas. The arm representation appeared to have extended laterally into the deafferented hand region. However, unlike the hand-face border, it is hard to clearly establish the prelesion hand-arm boundary, precluding a measurement of the exact extent of the expansion. As in normal monkeys, none of the recordings produced receptive fields on both the arm and the hand.

More medially, the leg, the trunk, and the foot were represented in the expected somatotopic order (Fig. 1) (Nelson et al., 1980). Presence of responses to touch on these body regions likely reflects the sparing of the fasciculus gracilis (Fig. 4B). No responses to touch on the face were found in this region of area $3 \mathrm{~b}$.

Finally, tactile stimuli failed to elicit clear responses at a number of recording sites (Fig. 4, $\mathrm{x}$ ). This could reflect the deafferentation, but most likely most of the unresponsive sites were a result of the site being suboptimal relative to the cortical layers during the long vertical electrode penetrations (Fig. 2C). Unresponsive sites were found in the face and the lower body portions of area $3 \mathrm{~b}$, regions expected to have normal or near normal sensory activation, and they commonly occur in such electrode penetrations in normal monkeys.

Monkey MCh73. In monkey MCh73, which was mapped 22 months after the lesion, the dorsal column lesion was at C7/C8 level (Fig. 9C). The lesion completely damaged the dorsal columns on the left side except for very minor sparing of the dorsomedial part corresponding to part of the gracilis fasciculus (Fig. 9B). There was also some damage to the contralateral dorsal columns adjacent to the midline. The ipsilateral dorsal horn was also damaged. This resulted in complete sparing of inputs from the radial hand and arm and some from the ulnar hand (Sherrington, 1939; Dykes and Terzis, 1981; Florence et al., 1988, 1989; Dykes et al., 1995).

In this monkey, we observed an expansion of the face representation into the hand region up to a distance of $\sim 7.5 \mathrm{~mm}$ medial to the normal hand-face border, which was determined using myelin-stained sections of the flattened cortex (see below). The region of the cortex where we found abnormal responses to touch on the face was more limited compared with MCh75. The expanded face responsive region was not contiguous but was found in four separate patches, only two of which bordered the normal face region. All of the face responsive recording sites found in the reorganized hand region had dual receptive fields on both the face and the hand. Out of a total of 174 recording sites, there were 56 sites with receptive fields on both the face and the hand. Unlike monkey MCh75 there was no region in the hand cortex where neurons responded only to the stimulation of the face. As for monkey MCh75 the receptive fields in the expanded face representation were confined to the chin (Fig. $10 \mathrm{~B}$ ) whereas those in the normal face region were present all over the face (Fig. 
$10 A)$. Neurons at the remaining 118 recording sites in the hand region had receptive fields on the hand alone.

Consistent with the level of the lesion, the hand region in monkey MCh73 had normal topography for the radial half of the hand (Fig. 11). Borders between digits D1-D2, and D2-D3 were at the normal, expected locations. This was confirmed by comparing with the anatomical borders between these digits revealed in the sections of the flattened cortex stained for myelin (Fig. 11, compare A, B). Receptive fields were small, restricted to a small portion of the skin of the digit and became smaller toward the distal part of the digits as in normal monkeys (Fig. 11C) (Nelson et al., 1980). The neurons responded to light cutaneous touch on the hand. In the medial portion of the hand representation, we encountered neurons responding to stimulation on D4 and D5. However, parts of these inputs are expected to be disrupted by the lesion. Consistent with this expectation, the somatotopy in this region was abnormal. The border between the D4 and D5 representations was not apparent (cf. Nelson et al., 1980), and the receptive fields tended to be larger, covering entire digits or spread over two digits (Fig. 11D). The neurons in this region generally responded to taps rather than cutaneous touch, similar to the hand representation in area $3 \mathrm{~b}$ of monkey MCh75.

In the myelin-stained sections cut tangential to the cortical surface from this monkey, a septum corresponding to the normal hand face border was apparent, as well as septa between representations of digits 1 and 2, and digits 2 and 3 (Fig. $11 A$ ). The recording results were consistent with the interpretation that the face, and digits 1 and 2 were represented in normal locations (Fig. $11 B$ ), but inputs from the face coactivated parts of the hand representation (Fig. 9).

Monkey MCh21. The monkey MCh21, which was mapped 23 months after the lesion, had the most extensive expansion of the face inputs. The lesion was at the level of C5 (Fig. 12C). It completely cut the dorsal columns and damaged part of the contralateral fasciculus gracilis (Fig. 12B). There was also damage to the ipsilateral dorsal horn. However, in this case the lesion extended to the ipsilateral ventral horn and part of the white matter at the junction of the ventral and lateral funiculi as evidenced by the presence of translucent scar tissue, which is likely because of secondary damage after the lesion (Fleming et al., 2006). Neurons at all the sites mapped in the expected hand representation had responses to stimulation on the face (Fig. 12A) with the receptive fields located on the chin (Fig. 13B). At two sites that bordered the normal face representation the receptive fields extended to the upper lip (Fig. 13B, left most figurine). There was only one recording site where we found neurons responding weakly to taps on D5. Neurons at this site also responded to stimulation on the chin. For this monkey, the cortex was sectioned in an off- parasagittal plane. Thus, the septum that marks the normal hand-face border was not apparent. However, the border between the normal hand and face representations is known to be near the end of the intraparietal sulcus (Florence and Kaas, 1995) (see Fig. 15). In area $3 \mathrm{~b}$ lateral to the end of the intraparietal sulcus, the receptive fields on the face were located all over the face (Fig. 13A) as in normal monkeys.

At more medial recording sites, as in monkey MCh75, we found neurons responding to stimulation of both arm and face. We mapped a total of 25 sites with responses to stimulation on the arm. Neurons at 16 of these sites had dual receptive fields on the arm and the chin. All the receptive fields on the arm were on the anterior skin of the upper arm, inputs from which enter the spinal cord rostral to the site of the lesion (Fig. 14). Unlike the other two monkeys, we also found neurons that responded only to the stimulation of the chin in the expected arm region of cortex. There was also an expansion of the arm representation. The arm representation extended $\sim 4 \mathrm{~mm}$ mediolaterally, which is approximately twice the width reported in normal monkeys 

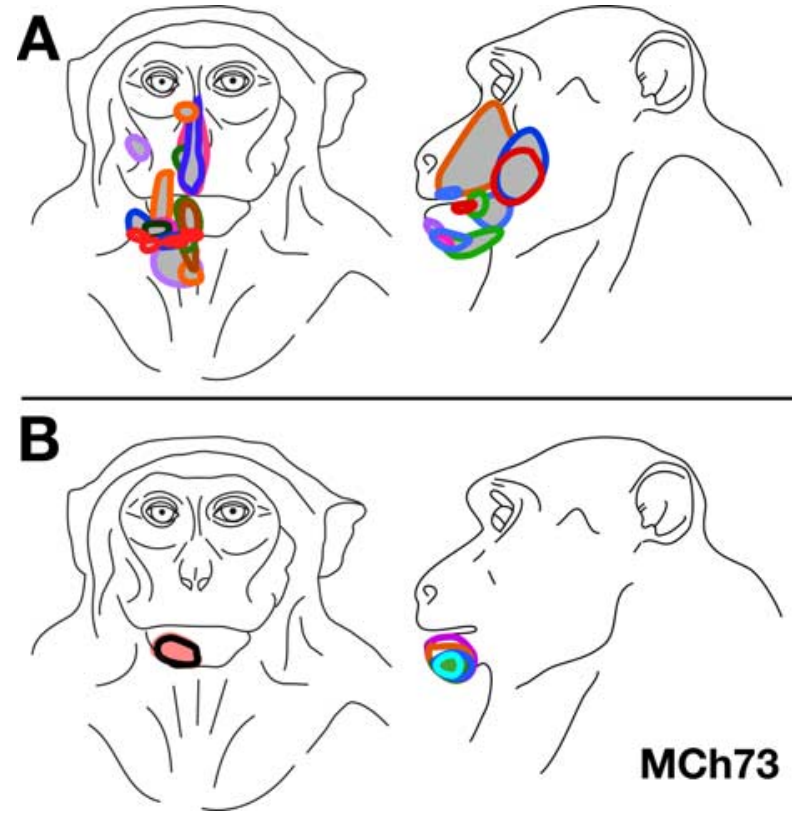

Figure 10. A, Locations of all the receptive fields on the face that were found in the normal face region lateral to the hand-face border in monkey MCh73 (Fig. 9A). Note that the receptive fields are located all over the face. $\boldsymbol{B}$, Locations of all the receptive fields on the face that were found in the reorganized cortex, medial to the hand-face border in monkey MCh75 (Fig. 9A). Note that all the receptive fields are confined to the chin.

(Nelson et al., 1980). However, in the absence of any physical landmark for the hand-arm border it was not possible to precisely determine the extent to which the arm representation expanded laterally into the hand region.

Medial to the hand representation, we encountered the shoulder/arm/occiput region, the inputs that enter the spinal cord rostral to the lesion (Fig. 14) (Sherrington, 1939; Nelson et al., 1980). Interestingly, even at these very medial locations we found neurons responding to stimulation on the chin. There were neurons that responded to stimulation on the chin, occiput and arm, and those that responded to stimulation on the dorsal upper trunk, chin and arm; that is, receptive fields were located on three different body parts (Fig. 14). Although in normal monkeys, the shoulder/occiput/neck region and the trunk region are located at the same mediolateral level, the presence of neurons with receptive fields on these body parts intermingled with neurons with receptive fields on the arm has not been reported. Because the occiput region extends laterally up to the level of the single site where we encountered responses to D5, it appears that these representations have expanded laterally into the arm region (compare with Fig. 1).

More medially, trunk, leg and foot representations were found in the expected somatotopic order. However, we found that neurons in the medial-most region, next to and along the medial wall also responded to stimulation on the chin (Fig. 13C). We encountered neurons with dual receptive fields on the foot and the chin, as well as those with receptive fields on the chin alone. At a few points in this region, we also encountered neurons with responses to stimulation on parts of the occiput/shoulder/neck regions. We estimated that the expansion of the face inputs up to the occiput representation was as much as $10 \mathrm{~mm}$ from the normal hand-face border. However, because the expansion of the face inputs was up to the foot representation, the overall expansion was $>20 \mathrm{~mm}$.

It is unlikely that the presence of the chin inputs in the foot
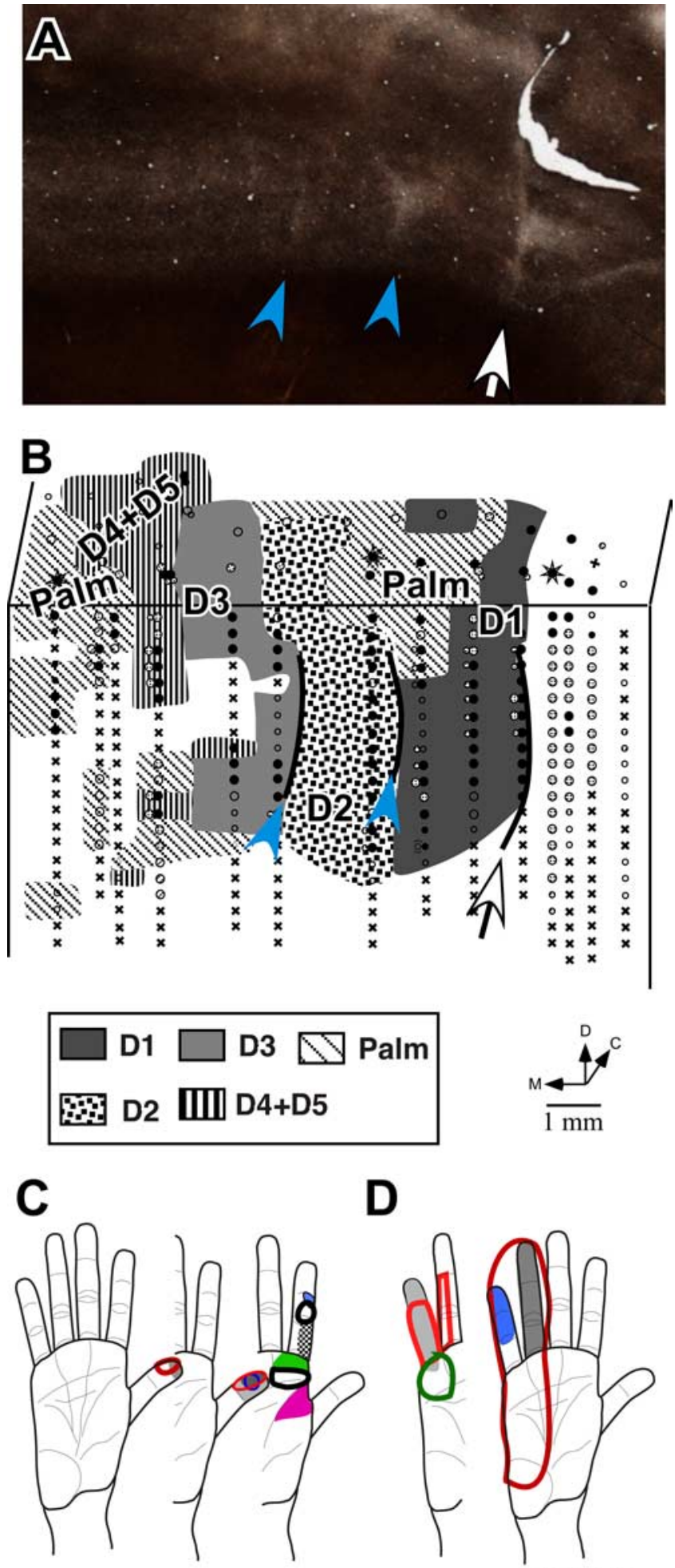

D

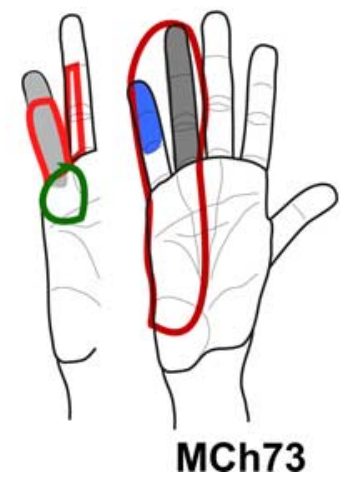

Figure 11. A, A myelin-stained section through area $3 b$ of the flattened cortex of monkey MCh73 cut parallel to the pial surface to reveal the hand-face border (white arrow) and the borders between digit 1 - digit 2 and digit 2-digit 3 (blue arrowheads). $\boldsymbol{B}$, Somatotopy of the hand in monkey MCh73. Although the progression of receptive fields in the lateral part (D1, D2, and radial palm) was normal, the medial part had no clear somatotopy and no clear borders between the representations of D4 and D5 digits. Conventions as for Figure 4A. C, Representative receptive fields on digit 1 , digit 2 , and radial palm for monkey $M C h 73$. Note that the receptive fields are small as for a normal monkey. $D$, Representative receptive fields on digit 4, digit 5, and ulnar palm for the same monkey. The receptive fields are large covering an entire digit or multiple digits. 

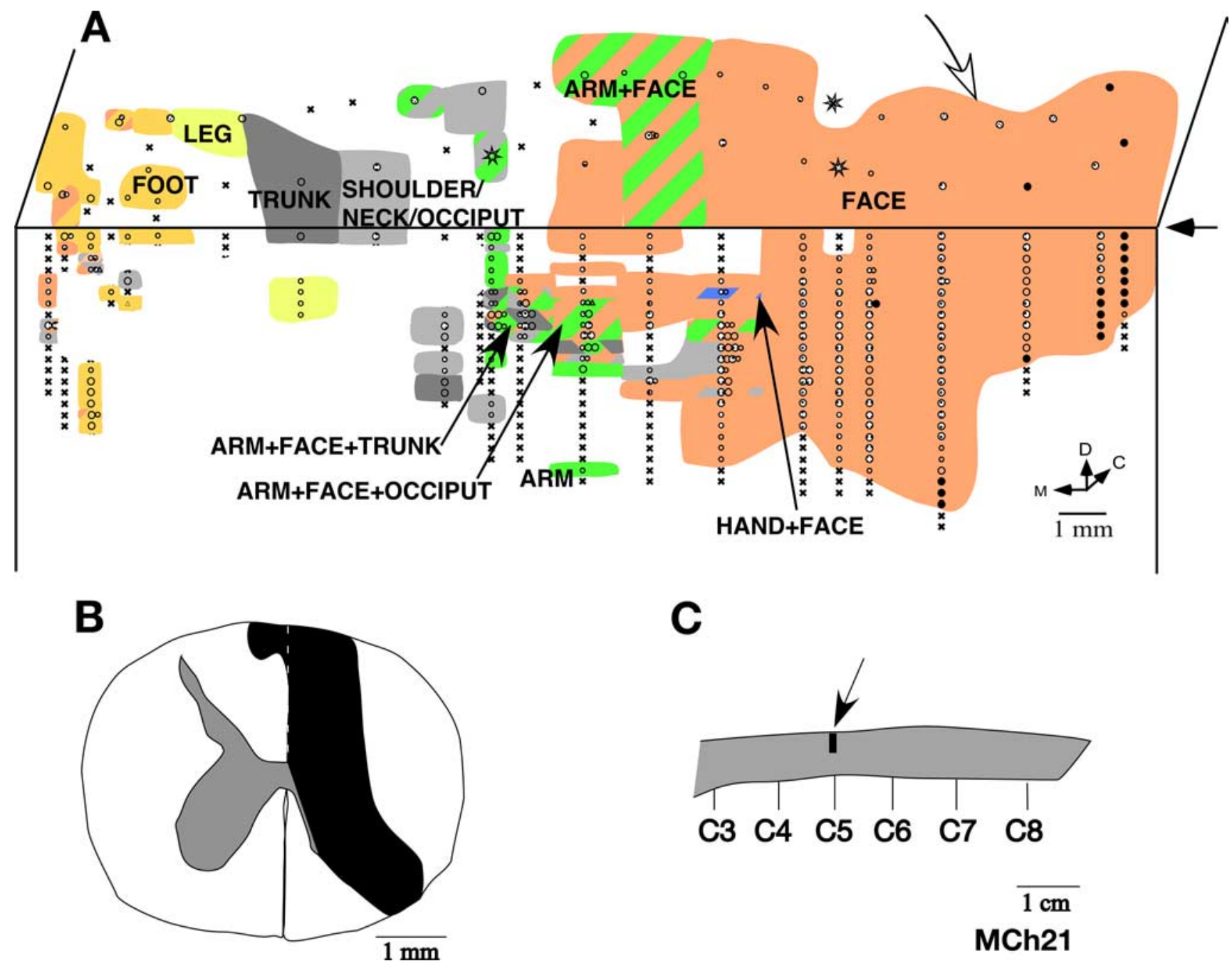

Figure 12. A, Somatotopy in the left area $3 \mathrm{~b}$ and the bordering cortex in monkey MCh21. The white arrow on the top marks the expected location of the normal hand-face border as estimated from the location of the tip of the intraparietal sulcus (Fig. 15). Note the expansion of the face responsive regions into the deafferented hand, arm and occiput areas. Receptive fields on the face were also seen in the medial most region intermingled with the leg and the foot representations. Conventions as for Figure $4 A$. $\boldsymbol{B}$, Reconstruction of the spinal cord lesion on a coronal section (region shown in black). C, Location of the lesion (arrow) on a dorsal view of the spinal cord. Rostral is to the left.

and leg region in monkey MCh21 is because of the lesion extending into the ventrolateral region of the spinal cord because (1) the influence of such a lesion on crossed spinothalamic pathways is likely to be in cortex ipsilateral to the lesion and (2) lesions of the ventrolateral spinal cord do not lead to the face region expanding into the foot region (Weng et al., 2000). However, it cannot be ruled out that the lesions of the ventrolateral pathway in combination with the dorsal column pathway lead to such reorganization.

\section{Immediate effects of dorsal column lesions on the organization of} area $3 b$

In two monkeys, MAc19 and MAc20, we did a limited mapping of area $3 \mathrm{~b}$, made a unilateral lesion of the dorsal columns and remapped at or near the previously mapped penetration sites. The goal of these experiments was to determine if neurons located in the normal hand region acquire receptive fields on the face immediately after the lesion.

Monkey MAc19. In monkey MAc19, we made one penetration in the region of the face representation and two penetrations in the adjacent hand region to establish the hand face border with a resolution of $\sim 800 \mu \mathrm{m}$ (Fig. 15C). Additionally, three penetra- tions were made in the hand area and one in the arm area. Dorsal columns contralateral to the mapped hemisphere were then lesioned. After the lesion, area $3 \mathrm{~b}$ was remapped (Fig. 15D) with the electrode at the same angle as before the lesion.

The dorsal column lesion was at the C5/C6 level. Reconstruction of the lesion site showed that the dorsal columns were completely cut except for a small sliver of tissue next to the midline. There was some damage to the ipsilateral dorsal horn (Fig. 15, bottom inset). For histological processing, the cortex of this monkey was flattened and series of sections were stained for cytochrome oxidase (Fig. 15A) or myelin (Fig. 15B) to confirm the electrode penetration sites and the hand-face border respectively. As expected, the prelesion electrode tracts that encountered neurons responsive to digit 1 were located between the septum at the histological hand-face border and the septum marking the D1-D2 border (Fig. 15B). Neurons responsive to the face were found lateral to the hand-face septum.

After lesion, we found no expansion of the face representation into the hand area. In the region of the hand representation, the neurons were unresponsive to stimulation of the skin of any part of the body. In one penetration adjacent to the face representation, we could evoke very weak responses to hard taps on the 

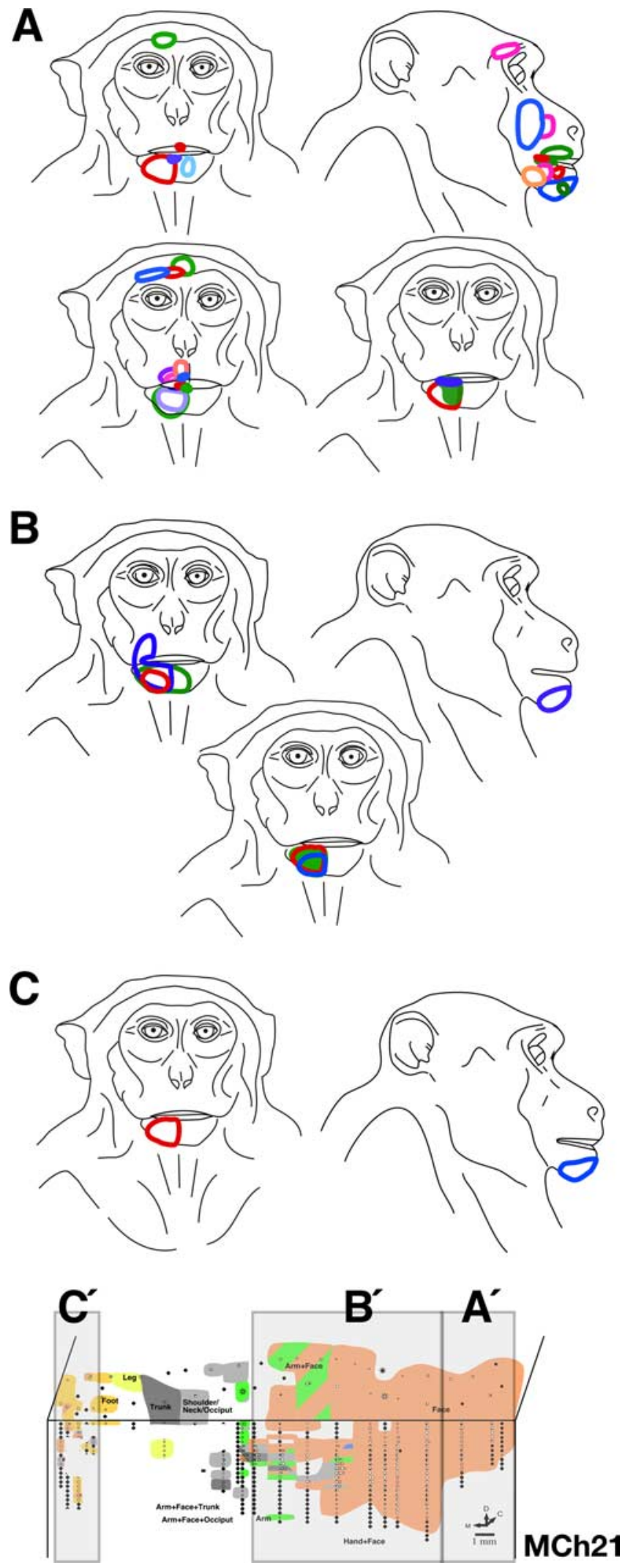

Figure 13. A, Locations of all the receptive fields on the face for monkey MCh21 that were observed in the normal face region lateral to the hand-face border. This region is marked as region $\boldsymbol{A}^{\prime}$ in the inset shown at the bottom (see Fig. 12A). The receptive fields are located all over the face as in a normal monkey. $\boldsymbol{B}$, Locations of all the receptive fields on the face that were found in the reorganized cortex medial to the hand-face border and lateral to the trunk representation marked as region $B^{\prime}$ inset shown at the bottom. Note that the receptive fields at all the recording sites are confined to the chin except for one receptive field that extends to the upper upper lip and cheek at depths between 5600-6400 $\mu \mathrm{m}$ from the surface. At a few points near the medial hand representation, we found weak responses to the taps or hard taps on the arm or ulnar digits and the palm, perhaps attributable to sparing of some of the fibers in the dorsal columns (Fig. 15).

Thus, there was no emergence of the face responses in the hand area immediately after the dorsal column lesion. Although it cannot be ruled out that some minor expansion of the face representation up to a distance of $<800 \mu \mathrm{m}$ could have occurred.

Monkey MAc20. The results from Monkey MAc20 were similar to those for the monkey MAc19. The lesion at the C5 level (Fig. $16 C$ ) was complete with additional damage to the columns on the contralateral side, next to the midline, which damaged mainly a part of the contralateral gracilis fasciculus (Fig. 16D). There was also some damage to the ipsilateral dorsal horn. Area $3 \mathrm{~b}$ of this monkey was mapped immediately before (Fig. 16A) and after (Fig. 16B) a unilateral dorsal column lesion. Neurons in the hand region did not respond to stimulation of any part of the body except at a few locations where very weak responses to very hard taps on the hand were seen. The receptive fields could not be localized to any particular part of the hand. The small region of the arm representation that was mapped continued to show responses similar to the prelesion state except that the responses were somewhat weaker. There was no observable immediate expansion of the face representation into the deafferented hand region of area $3 b$.

\section{Reorganization of the ventroposterior nucleus of the thalamus}

In normal monkeys, somatic inputs to the VP nucleus of thalamus are organized in a somatotopic manner. Inputs from the face are medial most in VPM (the medial subdivision of the VP nucleus) followed by hand, foot and tail in successive lateral representations in VPL (the lateral subdivision of the VP nucleus). The arm, leg, occiput and trunk are represented in more dorsal locations (Fig. 3) (Jones and Friedman, 1982; Kaas et al., 1984). In the VP nucleus the regions that receive inputs from the face, hand, foot and tail can be distinguished in histological preparations. The borders between these representations are seen as cell sparse septa in sections stained for Nissl substance, and as lightly stained regions in the sections stained for cytochrome oxidase activity (Fig. 3). The locations of the neurons that were mapped in the present study were confirmed in histological preparations. We investigated whether the topographic arrangement in the VP nucleus undergoes reorganization after lesions of the dorsal columns, and how this reorganization compares with the reorganization in area $3 \mathrm{~b}$ of cortex.

Reorganization of the ventroposterior nucleus of the thalamus in monkeys with long-term dorsal column lesions

The ventroposterior nucleus of the thalamus was mapped in two monkeys with chronic lesions: MCh75 and MCh21. The somatosensory cortex (area 3b) was also mapped in the same two monkeys (Figs. 4, 12). We found that after lesions of the dorsal columns and long recovery periods, the VP nucleus of the thalamus shows topographic reorganization.

Monkey MCh75. In monkey MCh75, we made a total of 12 penetrations in the left ventroposterior nucleus, which is con-

$\leftarrow$

lip. C, Locations of all the receptive fields on the face that were found in the reorganized cortex medial to the trunk representation marked as region $\boldsymbol{C}^{\prime}$ inset shown at the bottom. The receptive fields at all the recording sites were confined to the chin as for the region $\boldsymbol{B}^{\prime}$. 
tralateral to the dorsal column lesion (Fig. $4 B, C)$. In seven penetrations, we encountered neurons with responses to touch on various body parts. In the medial most penetrations \#114, \#115, and \#116 (Fig. $17 B$ ), which passed through VPM and the parvocellular subdivision of the VP nucleus (VPMpc), we encountered neurons with receptive fields on the face and in the oral cavity as expected (receptive fields not shown). However, receptive fields of neurons in more lateral penetrations did not match with the expected thalamic somatotopy or the architecture. In two of the penetrations, which passed through the hand subnucleus of the VPL (Fig. 17A, pen \#119, $C$, pen \#120) we encountered neurons responding to stimulation of the chin where responses to the touch on the hand were expected. At most of these locations, neurons responded to touch on both the hand and chin as was observed for area $3 b$ for this monkey (Figs. 4, 7). In addition, in penetrations \#118, \#119, and \#120, we found neurons responding to stimulation on the arm at locations which are more ventral than in normal monkeys and correspond to locations of the inputs from the hand (Jones and Friedman, 1982; Kaas et al., 1984). This expansion of the arm representation into the hand region is similar to the lateral expansion of the armresponsive region into the hand region in area 3b (Fig. 4). At more superficial levels in these penetrations, we encountered neurons with receptive fields on the arm, and the occiput/neck area as expected.

Monkey MCh21. In the VP nucleus of monkey MCh21, which was mapped 23 months after a complete lesion of the dorsal columns, we found a more extensive lateral shift in the representation of the chin. A total of 19 penetrations were made through the VP nucleus (Figs. 1822). Neurons in VPM responded to stimulation on the face and oral cavity with receptive fields distributed all over the face, as expected. Somatotopy was normal in VPM (Fig. 19, penetration $\# 122$, Fig. 21, part of \#120). In contrast, the somatotopy in VPL was highly abnormal. We did not find any receptive fields on the deafferented regions of the body, that is on the contralateral hand, forearm, or the deafferented parts of the upper arm. At three recording sites in penetrations \#110 (Fig. 20) and \#126 (Fig. 18), we encountered responses to stimulation of the hand. At all these sites, the neuronal responses were weak and could only be elicited after stimulation of the deep tissue, and it was not possible to precisely localize the receptive fields
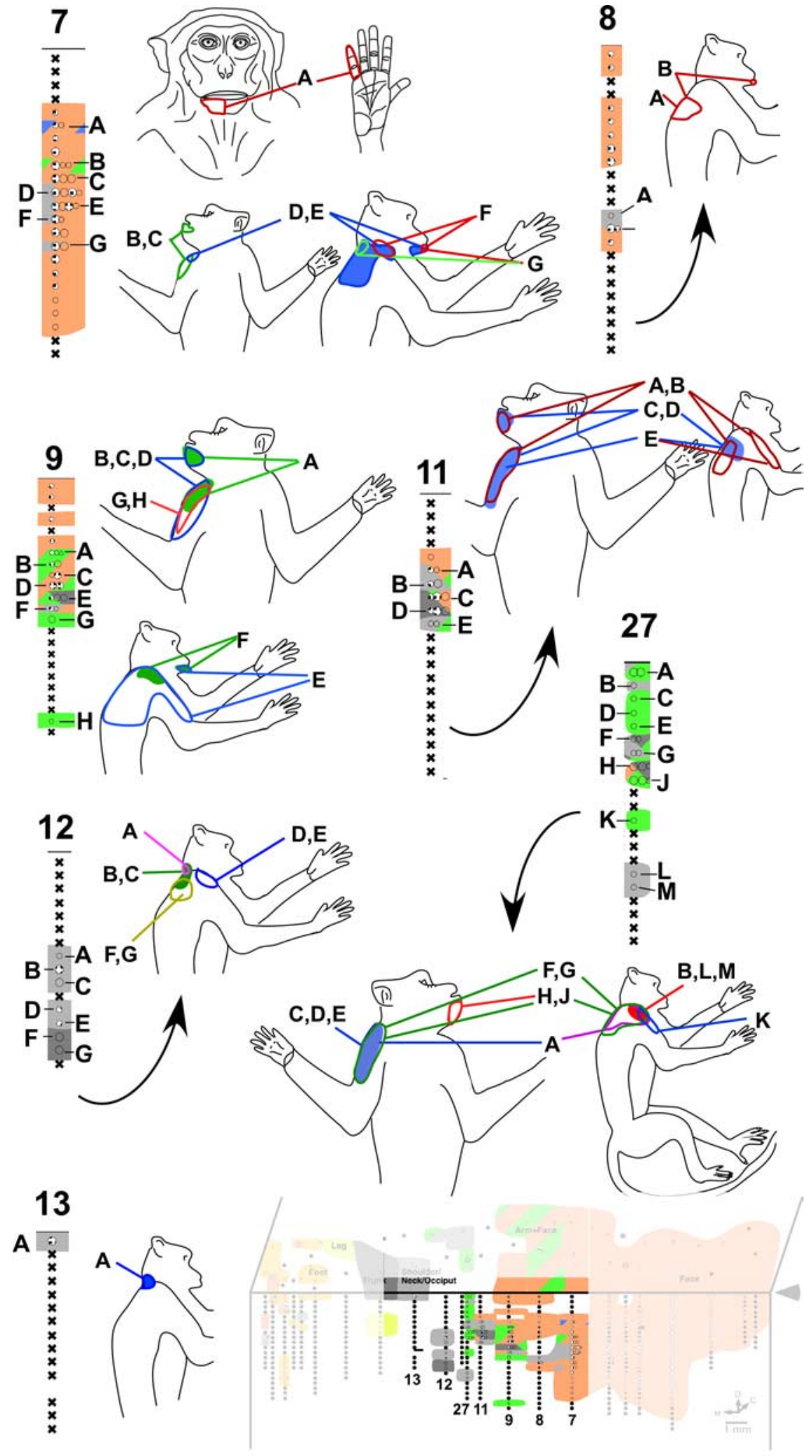

MCh21

Figure 14. Receptive fields of neurons in penetrations through area $3 \mathrm{~b}$ in the region where in addition to receptive fields on the chin we encountered neurons with responses to touch on the hand, arm, or occiput. The receptive fields of neurons in each penetration at the sites marked by letters are shown on the adjacent figurines. Note that the receptive fields on the arm were confined to the anterior upper arm. Only at one site (site " $\mathrm{A}$ " in penetration 7) did neurons respond to touch on the hand (digit 5). The locations of the electrode penetrations are shown on the inset on the bottom right (compare Fig. 12A). 

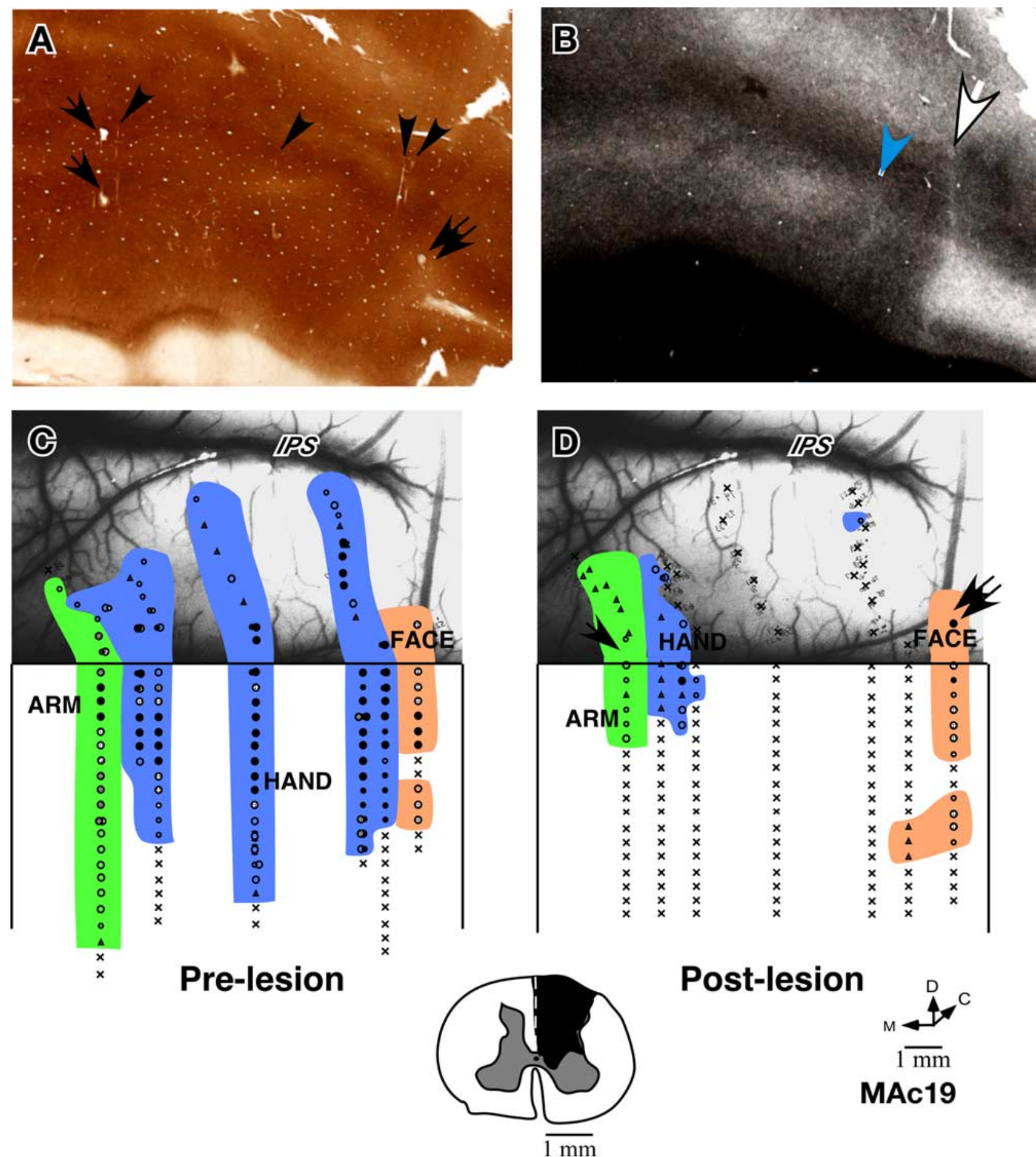

Post-lesion
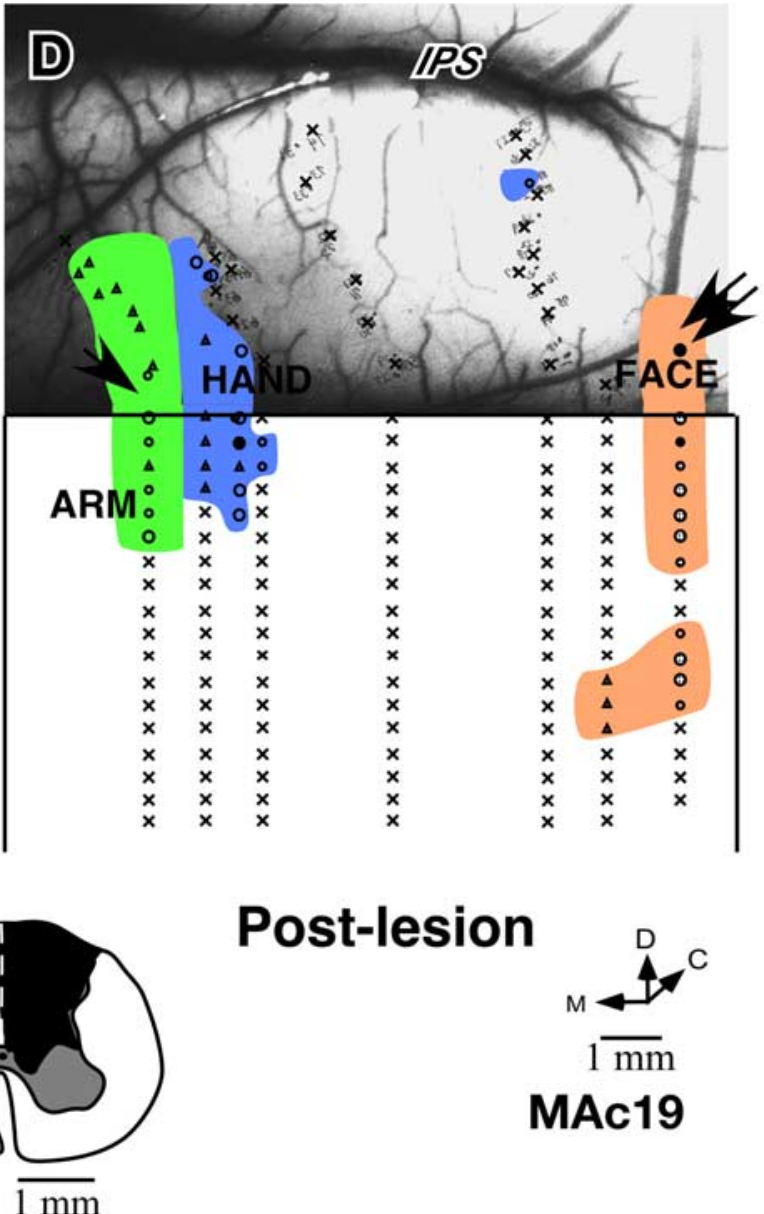

Figure 15. Somatotopy in area $3 \mathrm{~b}$ of monkey MAc19 before and immediately after lesion of the dorsal columns. $A$, Cytochrome oxidase-stained section of the flattened cortex through area $3 \mathrm{~b}$ showing electrolytic microlesions made during the experiment (arrows and double arrow) and some visible electrode tracks (arrowheads). $\boldsymbol{B}$, A myelin-stained section of the flattened cortex showing the hand-face border (white arrow) and the border between D1 and D2 (blue arrowhead). C, Locations of neurons with receptive fields on the face, hand and arm encountered in area $3 \mathrm{~b}$ and the adjacent cortex before lesion of the dorsal columns of the spinal cord, and (D) immediately after lesion of the contralateral dorsal columns of the spinal cord. Only a few neurons responded to the taps on the hand or arm perhaps because of sparing of some of the dorsal column fibers. The arrow and the double arrow shown in D indicate the penetrations marked with electrolytic lesions (compare with $\boldsymbol{A}$ ). The photograph underlay in $\boldsymbol{C}$ and $\boldsymbol{D}$ shows the correspondence of the prelesion and postlesion penetration sites with respect to the surface vasculature of the brain. Intraparietal sulcus (IPS) is visible at the top of the photograph. Note that the hand-face border is located near the end of the IPS, our basis for interpretation of this border for monkey MCh21. Inset at the bottom shows the spinal cord lesion reconstructed from the transverse sections of the spinal cord. Conventions as for Figure $4 \mathrm{~A}$.

on the hand. The abnormal features of the map can be summarized as follows: (1) The arm representation was greatly expanded to include neurons down to the ventral-most regions of the ventroposterior nucleus. (2) There was an extensive expansion of representation of the inputs from the occi- put, shoulder and neck regions throughout VPL. (3) There were multiple receptive fields on the arm, chin and the occi$\mathrm{put} /$ neck region for neurons distributed throughout the reorganized VP nucleus. (4) There were multiple receptive fields with neural responses evoked from the face, leg and foot (Fig. 


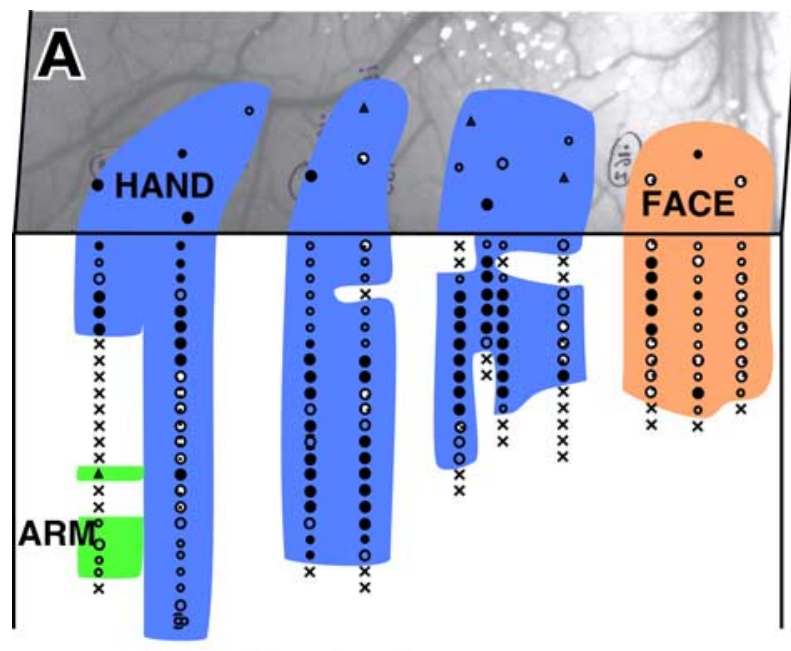

Pre-lesion

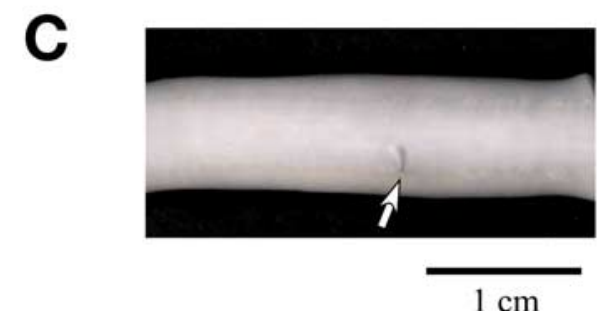

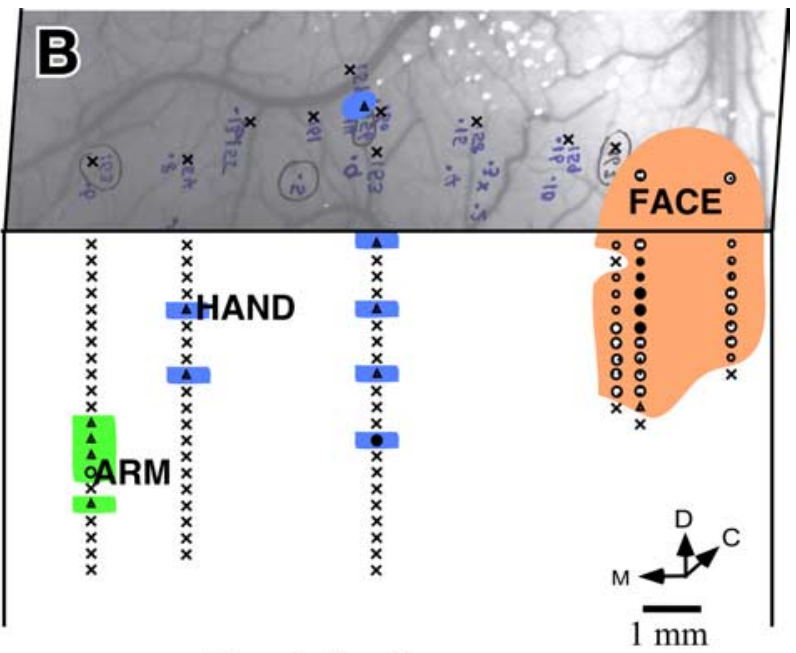

Post-lesion

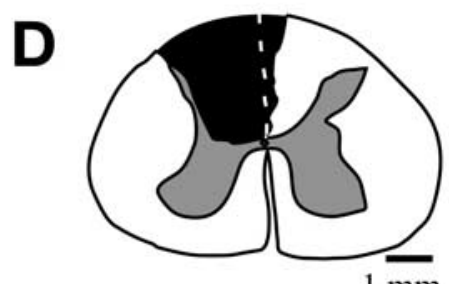

$1 \mathrm{~mm}$
MAc20

Figure 16. Somatotopy in area 3b of monkey MAc20 before and immediately after lesion of the dorsal columns. $\boldsymbol{A}$, Locations of neurons with receptive fields on the face, hand and arm encountered in area $3 \mathrm{~b}$ and adjacent cortex before, and $(\boldsymbol{B})$ immediately after lesion of the contralateral dorsal columns of the spinal cord. After lesion, only a few neurons responded weakly to hard taps on the hand or arm. The photograph underlay in $\boldsymbol{A}$ and $\boldsymbol{B}$ shows the correspondence of the prelesion and postlesion penetration sites with respect to the surface vasculature of the brain. $\boldsymbol{C}$ Photograph showing lesion (arrow) on the dorsal surface of the spinal cord. Rostral is to the left. $\boldsymbol{D}$, The lesion reconstructed from the transverse sections of the spinal cord.

18 , penetration \#104). There were also discrete receptive fields on the face as well as foot for recording sites in the same vertical penetration (Fig. 21, penetration \#113) unlike in normal monkeys. (5) The trigeminal face receptive fields that were abnormally located were all found on the chin. All these features were also seen in the reorganized area $3 \mathrm{~b}$ of the cortex (Figs. 12, 13), including presence of responses to stimulation of the occiput and the face in the foot/leg representations.

Comparing the normal histological borders between the foot, hand, and the face regions of the VP nucleus (see above) with the microelectrode recording results, it was clear that the reorganization in the VP nucleus did not include expansion of the remaining foot inputs into the adjacent deafferented hand subnucleus. However regions that are dorsal to the hand subnucleus, the arm and the occiput/neck/shoulder region did show an expansion into the hand region. The major source of reactivation in the deafferented parts of the hand and foot subnuclei was from tactile receptors on the chin.

\section{Reorganization of the ventroposterior nucleus immediately after} the dorsal column lesion

Monkey MAc20. In one monkey we made penetrations through the VP nucleus in the region of the face and the hand representations, lesioned the contralateral dorsal columns (Fig. 16) and immediately remapped approximately the same sites in the VP nucleus (Fig. 23). Postlesion responses to the stimulation of the skin of the hand or arm in the VPL were abolished (compare prelesion penetration \# 19 and \#20 with postlesion penetration $\# 101$ and \#103 respectively). Responses to the stimulation of the face remained normal in the VPM. We did not observe any ex- pansion of the face-responsive region into the hand area immediately after the dorsal column lesion.

\section{Discussion}

In the present study, we extensively mapped the somatosensory area $3 \mathrm{~b}$ and the VP nucleus of the thalamus of adult macaque monkeys after long-term recoveries or immediately after unilateral lesions of the dorsal columns at cervical levels. The major findings are: (1) the reorganization leading to the expansion of the face region in area $3 \mathrm{~b}$ is not restricted to the adjacent hand area but extends into the arm, occiput and distant foot regions; (2) there is lateral expansion of the arm and occiput region into the deafferented hand cortex; (3) the pattern of the large-scale reorganization is similar in the VP nucleus, including expansion of the face inputs into the foot region, the expansion of the arm into the hand subnucleus, and the expansion of the occiput/neck/ shoulder region into the hand and the foot regions. We also show that there is no immediate large-scale reorganization in either the VP nucleus or area $3 \mathrm{~b}$ after lesions of the dorsal columns.

\section{Limits of plasticity in area $3 b$}

The maximal extent of shift in representational boundaries reported before this study is in the range of $10-14 \mathrm{~mm}$ for macaque monkeys (Pons et al., 1991) and $~ 5 \mathrm{~mm}$ for smaller brained owl monkeys (Jain et al., 1997). This expansion is 1.7 times the mediolateral extent of the hand representation in area $3 \mathrm{~b}$ of adult macaque monkeys and a comparable 1.5 times for adult owl monkeys. Here we show that brain reorganization can extend well beyond these limits. The face representation can reach as far 

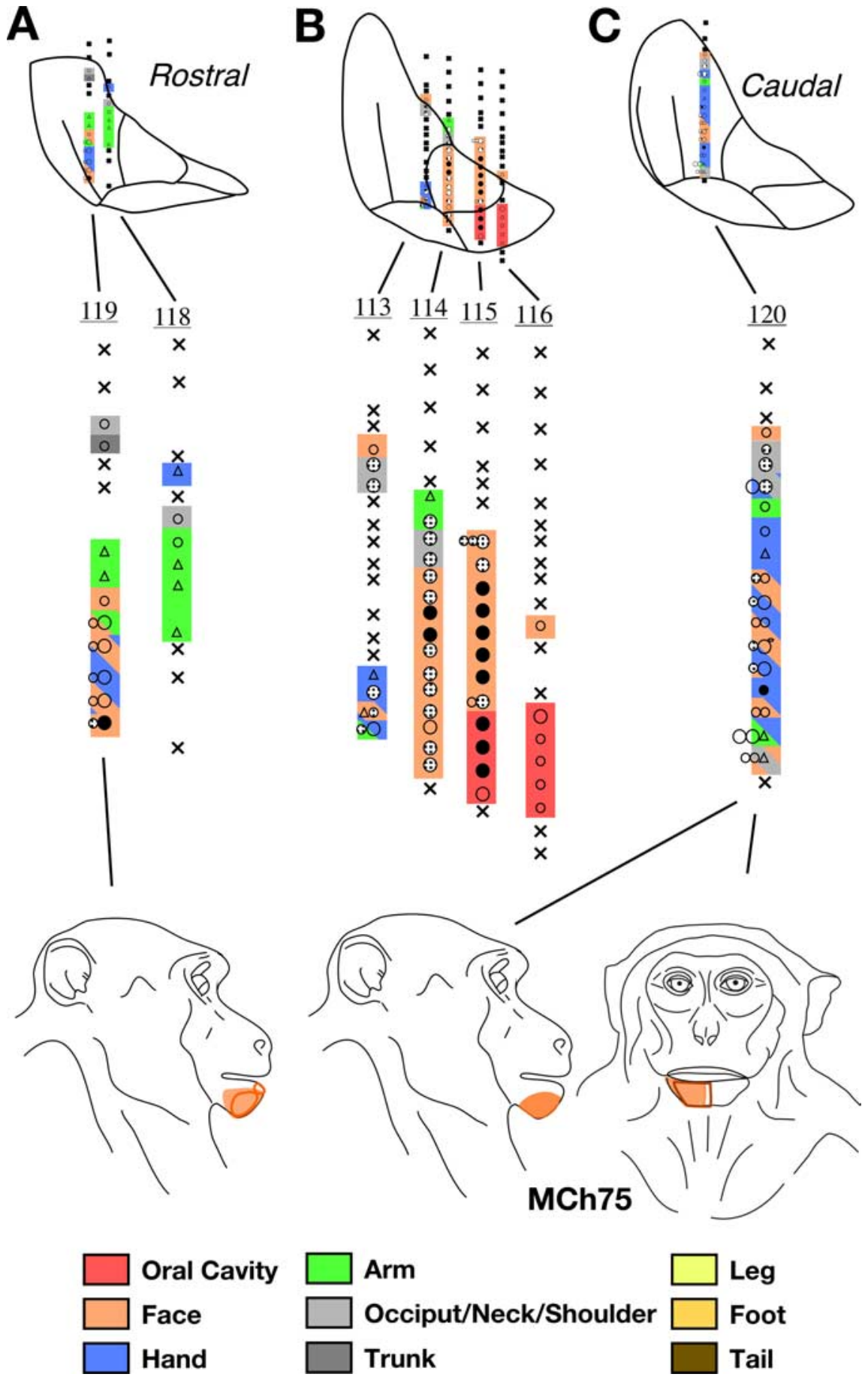

Figure 17. $\quad \boldsymbol{A}, \boldsymbol{B}, \boldsymbol{C}$, Somatotopy and receptive fields encountered in seven electrode penetrations through the VP nucleus of monkey MCh75 shown on a series of three rostral to caudal coronal sections. The figurines at the bottom show abnormally located receptive fields on the face for neurons in the lateral portion of VP. Normally located receptive fields, i.e., those on the face in VPM, and other body parts in VPL are not shown. For illustration purposes, electrode penetrations within a rostrocaudal extent of 500 $\mu \mathrm{m}$ are projected on a single intermediate section. The penetrations are numbered. The color key shown at the bottom also applies to the maps shown in Figures 18-23 and is same as used for illustrating maps of area 3b. The regions shown in double or triple colors by use of colored slanting lines indicate mapping sites where neurons had multiple receptive fields on body parts indicated by the colors of the lines. For other conventions, see Figure $4 \mathrm{~A}$.

as the medial most zone of area $3 \mathrm{~b}$, which is up to $20 \mathrm{~mm}$ from the normal face representation as was observed in our one monkey with complete lesion. We have not come across any report that describes brain reorganization of this magnitude in adult primates. It is possible that this has not been reported before because the foot region of the cortex was not mapped previously (Pons et al., 1991; Jain et al., 1997).

\section{Nature of reorganization after} deafferentation in adult primates

Many kinds of deafferentations lead to an expansion of the adjacent inputs from the same body part into the deprived region of area $3 \mathrm{~b}$ and the VP nucleus of the thalamus. Following partial loss of hand inputs because of nerve cut or digit amputations there is "filling in" by the remaining hand inputs (Merzenich et al., 1983b, 1984; Wall et al., 1986; Garraghty et al., 1994); and after upper or lower limb amputations, representation of the skin of the limb proximal to the amputation expands (Lenz et al., 1998; Dostrovsky, 1999; Florence et al., 2000). In contrast, after transection of the dorsal roots of the spinal cord from C2 to T4, the deprived hand, arm, and occiput regions of area $3 \mathrm{~b}$ come to respond to the inputs from the chin (Pons et al., 1991). Similar changes also took place in the VP nucleus, although they were accompanied by gross morphological changes, attributed to transneuronal degenerations (Jones and Pons, 1998). Our results presented here and those previously from owl monkeys (Jain et al., 1997) show reorganization that involves both the expansion of the face inputs as well as any remaining hand inputs in both area $3 \mathrm{~b}$ and VP nucleus.

Previous reports of expansions of the preserved inputs in VP nucleus after spinal cord injuries relate to deprivation of the lower body. Pollin and Albe-Fessard (1979) reported a lateral shift of the hand representation in the foot region of the VP nucleus 2-4 weeks after dorsal quadrant lesions at T3-T5 levels. In patients with spinal cord injuries at thoracic levels there was an expansion of the body region just proximal to the deafferented part (Lenz et al., 1994). However, we are not aware of any previous reports of expansion of the face into the lower limb region in the VP nucleus.

Our results also show that there is an expansion of a third set of intact inputs into the deafferented regions of area $3 \mathrm{~b}$ and the VP nucleus. These inputs originate from part of the anterior arm and the occiput regions. Likewise, in a human patient with spinal lesion at the C6 level, a great portion of the expected hand region of the VP nucleus was occupied by the representation of the neck, occiput and ear (Lenz et al., 1987). In our experiments, the occiput region expanded into the hand and the foot subnuclei of VP as well as these representations in the cortex, whereas the arm representation expanded only in the hand subnucleus. 
It is not clear why we do not see an expansion of the foot inputs into the hand area particularly in the VP nucleus of thalamus because these representations are also adjacent. In the somatosensory cortex of a human with amelia who was missing both upper limbs since birth, MEG studies showed that both the face and the foot representations expanded toward the hand region, although the foot region may not have moved lateral enough to be in the normal hand region. Thus, an expansion of the foot region appears to be possible. In another study, in the post central cortex, a larger than normal activation was seen on stimulation of the stump in a person with lower leg amputation (Condés-Lara et al., 2000). However, here also it is not clear if the stump representation expanded beyond the region of the leg area into the representation of a different body part. As another example of restricted expansions, after median nerve cut only radial nerve inputs expand into the deprived region of area $3 \mathrm{~b}$ although the deprived median nerve region lies immediately adjacent to the normally innervated face region (Merzenich et al., 1983a; Wall et al., 1983; Garraghty and Kaas, 1991b). Thus, there appear to be certain limitations to the representations that can expand into the deprived territories.

Correlation of the reorganization in the VP nucleus with cortical reorganization We observed an expansion of the chin, upper arm and the occiput region after dorsal column injuries in both area $3 \mathrm{~b}$ and the VP nucleus. Although the nature of reorganization in these two regions was similar, there were clear differences. For example, the occiput representation expanded throughout the hand subnucleus of the VP nucleus. Whereas in the somatosensory cortex neuronal responses to touch on the occiput region were restricted to the medial portion of the hand representation suggesting that reorganization in the VP nucleus may not be expressed completely in the cortex. Although previous reports from monkeys suggest mostly similar reorganizations in the $\mathrm{VP}$ nucleus and area 3b (Jones, 1983, 2000; Garraghty and Kaas, 1991a; Jones and Pons, 1998) differences have been reported (Florence et al., 2001), which could be because of shaping of the receptive fields at each level by complex interactions of the divergent feedforward and feedback connections (Ergenzinger et al., 1998; Krupa et al., 1999).

\section{Mechanisms of reorganization}

We did not observe large-scale plasticity in either area $3 \mathrm{~b}$ or the VP nucleus of thalamus immediately after lesion, although an

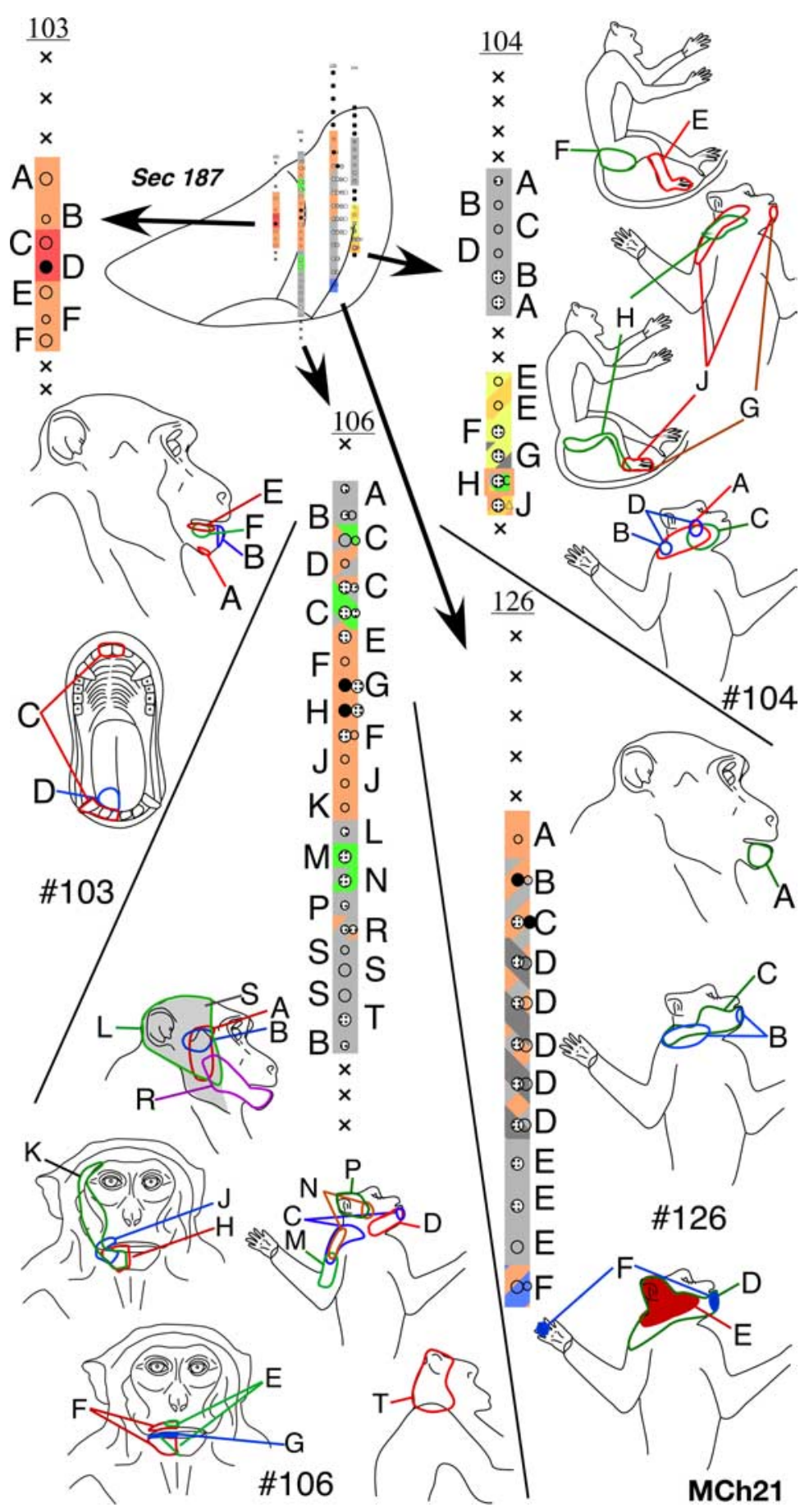

Figure 18. Somatotopy and receptive fields encountered in electrode penetrations through the ventroposterior nucleus of monkey MCh21 shown on a series of caudal to rostral sections in Figures 18-22. Penetrations within a rostrocaudal extent of 500 $\mu \mathrm{m}$ are projected on a single intermediate section. Conventions as for Figures $4 \mathrm{~A}$ and 17.

expansion of the order of few hundred micrometers cannot be ruled out. Previous results have shown an immediate expansion of the representations of adjacent digits after amputation or denervations of a digit, attributed to disinhibition of the preexisting inputs (Calford and Tweedale, 1988, 1991). Our previous results on the time course of cortical reorganization after chronic dorsal column lesions indicate that remaining inputs from the hand, if any, first expand to activate the deprived hand cortex over the initial 2 months of recovery. This is followed by expansion of inputs from the face, which were extensive by $6-8$ months, but 


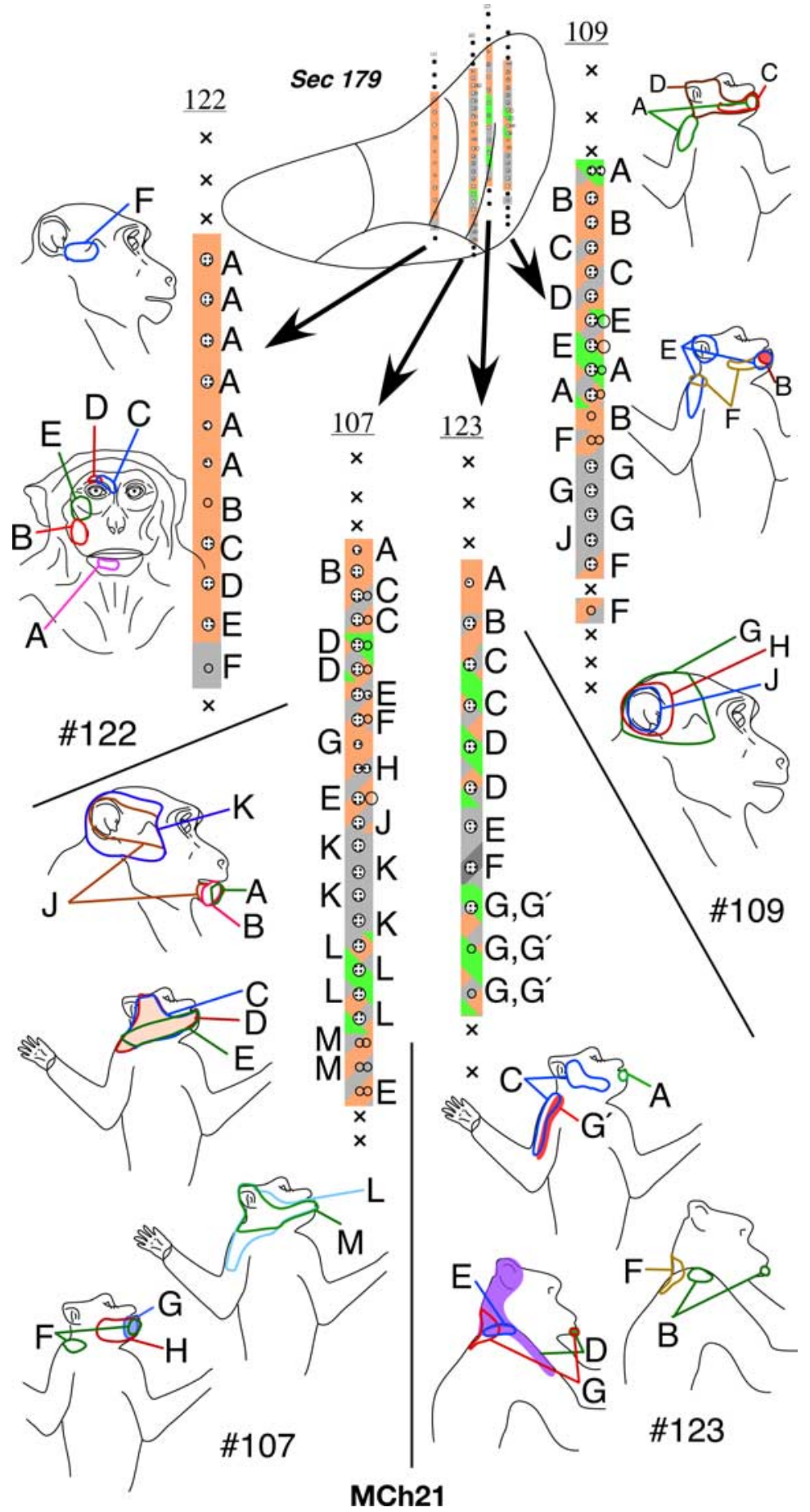

fore, the duration of the recovery period is unlikely to be the only factor.

A small expansion of the face inputs into the hand region of the cortex was seen long after hand or arm amputations (Flor et al., 1995; Florence and Kaas, 1995; Grüsser et al., 2004), or immediately after median and radial nerve injury or block (Silva et al., 1996; Weiss et al., 2004). Between the hand and the face representations in area $3 \mathrm{~b}$ of monkeys only few intracortical connections have been reported (Manger et al., 1997; Fang et al., 2002). Even these connections are restricted to a small region in the immediate vicinity of the hand-face border and would be insufficient to support the large-scale extensive reorganization observed here. However, it cannot be ruled out that minor changes in representational boundaries could be mediated by unmasking of the preexisting overlapping intracortical connections between the hand and the face representations (Manger et al., 1997), perhaps in combination with limited neuronal growth (Florence et al., 1998).

Reorganization in area $3 b$ of cortex does not follow the adjacency rule based on normal somatotopy, which would be essential if the overlap of thalamocortical connections was the only mechanism of plasticity. The expanded face region is not necessarily always contiguous with the normal face region (monkey MCh73) (Moore et al., 2000). In the monkey MCh21 with even more massive reorganization, the face representation expands into the leg region bypassing the entire trunk region of area $3 \mathrm{~b}$. Moreover, for expansion of intact face inputs a complete deafferentation of the adjacent cortex is not required. We saw an expansion of the face inputs even when a normal topography was preserved in the adjacent hand representation (monkey MCh73). Perhaps a partial injury is sufficient to induce reactive growth at brainstem, or thalamic levels leading to the reorganization (Jain et al., 2000).

The most likely site of growth is from the intact trigeminal nucleus in the brainstem to the completely or partially deaffer-

could have expanded between 2 months and 6 months (Jain et al., 1997). It is thus clear that preserved hand inputs, perhaps with overlapping silent connections, are the early candidates of plasticity, followed by changes that require other mechanisms such as more extensive neuronal growth (Jain et al., 2000). However, it is not clear why large-scale expansion of the face inputs into the deprived hand cortex has not been seen after deafferentation by cutting the nerves to the hand. The organization of the cortex after median nerve or median and ulnar nerve cuts has been explored after as much as five (Merzenich et al., 1983b; Garraghty and Kaas, 1991b) or 11 months (Garraghty et al., 1994). There- ented cuneate nucleus. The trigeminal inputs that expand have their receptive fields mostly centered on the chin, which in the trigeminal nucleus lie adjacent to the cuneate nucleus (Kaas et al., 1999). Although, in the VP nucleus of the thalamus the chin inputs are close to the hand region, no evidence of growth between these two regions of the VP nucleus is available. The notion of reactive growth of the chin inputs into the adjacent deprived hand region and further into the deprived foot region at the subcortical levels is attractive because such growth could take place bypassing the dorsal trunk representation, which in area $3 \mathrm{~b}$ is not activated by chin inputs in the monkeys with the lesions. 


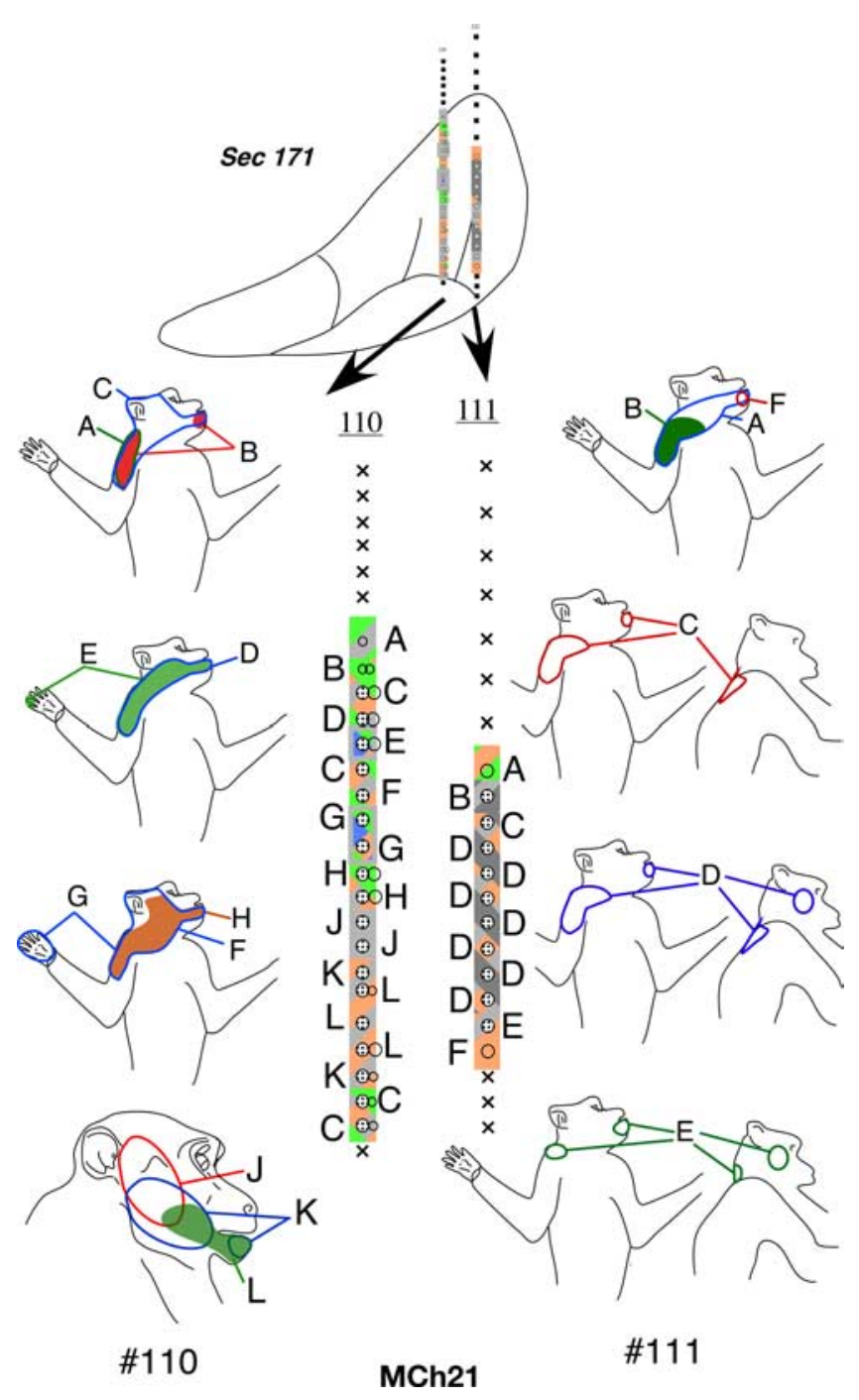

Figure 20. Please see legend to Figure 18.

Although the exact mechanisms of brain reorganization remain to be established, two interesting questions emerge. First, why does the foot representation not expand into the deafferented hand cortex of area $3 \mathrm{~b}$ or into the hand subnucleus of VP, whereas the chin representation can expand all the way medially into the foot representation? Second, why is the lateral expansion of the occiput and the arm representations rather limited, not extending further up to the hand-face border in area $3 \mathrm{~b}$ ?

\section{References}

Calford MB, Tweedale R (1988) Immediate and chronic changes in responses of somatosensory cortex in adult flying-fox after digit amputation. Nature 332:446-448.

Calford MB, Tweedale R (1991) Immediate expansion of receptive fields of neurons in area $3 \mathrm{~b}$ of macaque monkeys after digit denervation. Somatosens Mot Res 8:249-260.

Chen R, Cohen LG, Hallett M (2002) Nervous system reorganization following injury. Neuroscience 111:761-773.

Condés-Lara M, Barrios FA, Romo JR, Rojas R, Salgado P, Sánchez-Cortazar J (2000) Brain somatic representation of phantom and intact limb: a fMRI study case report. Eur J Pain 4:239-245.

Darian-Smith C, Brown S (2000) Functional changes at periphery and cortex following dorsal root lesions in adult monkeys. Nat Neurosci 3:476-481.

Darian-Smith C, Ciferri MM (2005) Loss and recovery of voluntary hand

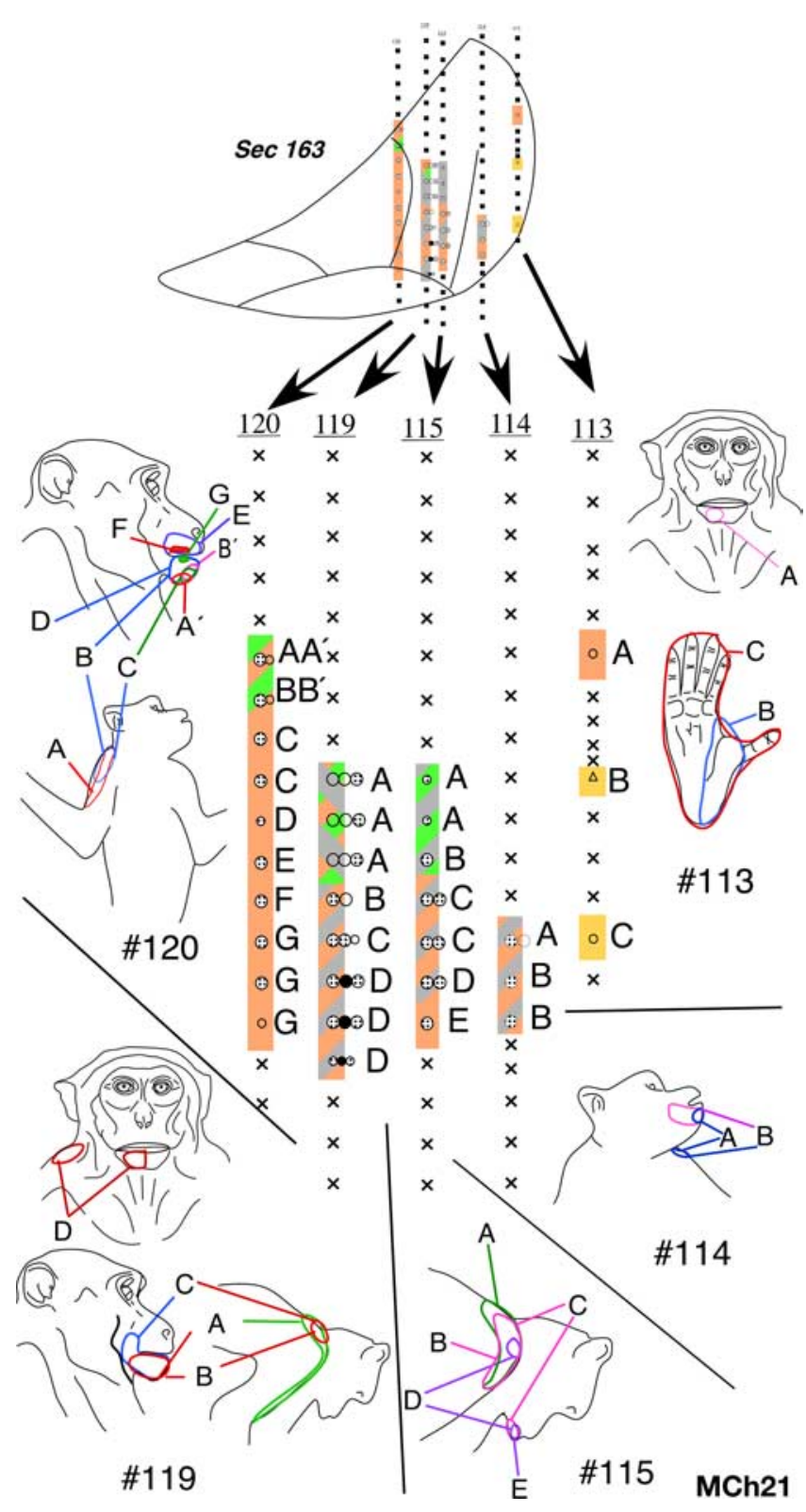

Figure 21. Please see legend to Figure 18.

movements in the macaque following a cervical dorsal rhizotomy. J Comp Neurol 491:27-45.

Dostrovsky JO (1999) Immediate and long-term plasticity in human somatosensory thalamus and its involvement in phantom limbs. Pain [Suppl 6]:S37-S43.

Dykes RW, Terzis JK (1981) Spinal nerve distributions in the upper limb: the organization of the dermatome and afferent myotome. Philos Trans R Soc Lond B Biol Sci 293:509-554.

Dykes RW, Avendaño C, Leclerc SS (1995) Evolution of cortical responsiveness subsequent to multiple forelimb nerve transections: an electrophysiological study in adult cat somatosensory cortex. J Comp Neurol 354:333-344.

Ergenzinger ER, Glasier MM, Hahm JO, Pons TP (1998) Cortically induced thalamic plasticity in the primate somatosensory system. Nat Neurosci $1: 226-229$.

Fang PC, Jain N, Kaas JH (2002) Few intrinsic connections cross the handface border of area $3 \mathrm{~b}$ of New World monkeys. J Comp Neurol 454:310-319.

Fleming JC, Norenberg MD, Ramsay DA, Dekaban GA, Marcillo AE, Saenz AD, Pasquale-Styles M, Dietrich WD, Weaver LC (2006) The cellular inflammatory response in human spinal cords after injury. Brain 129:3249-3269. 

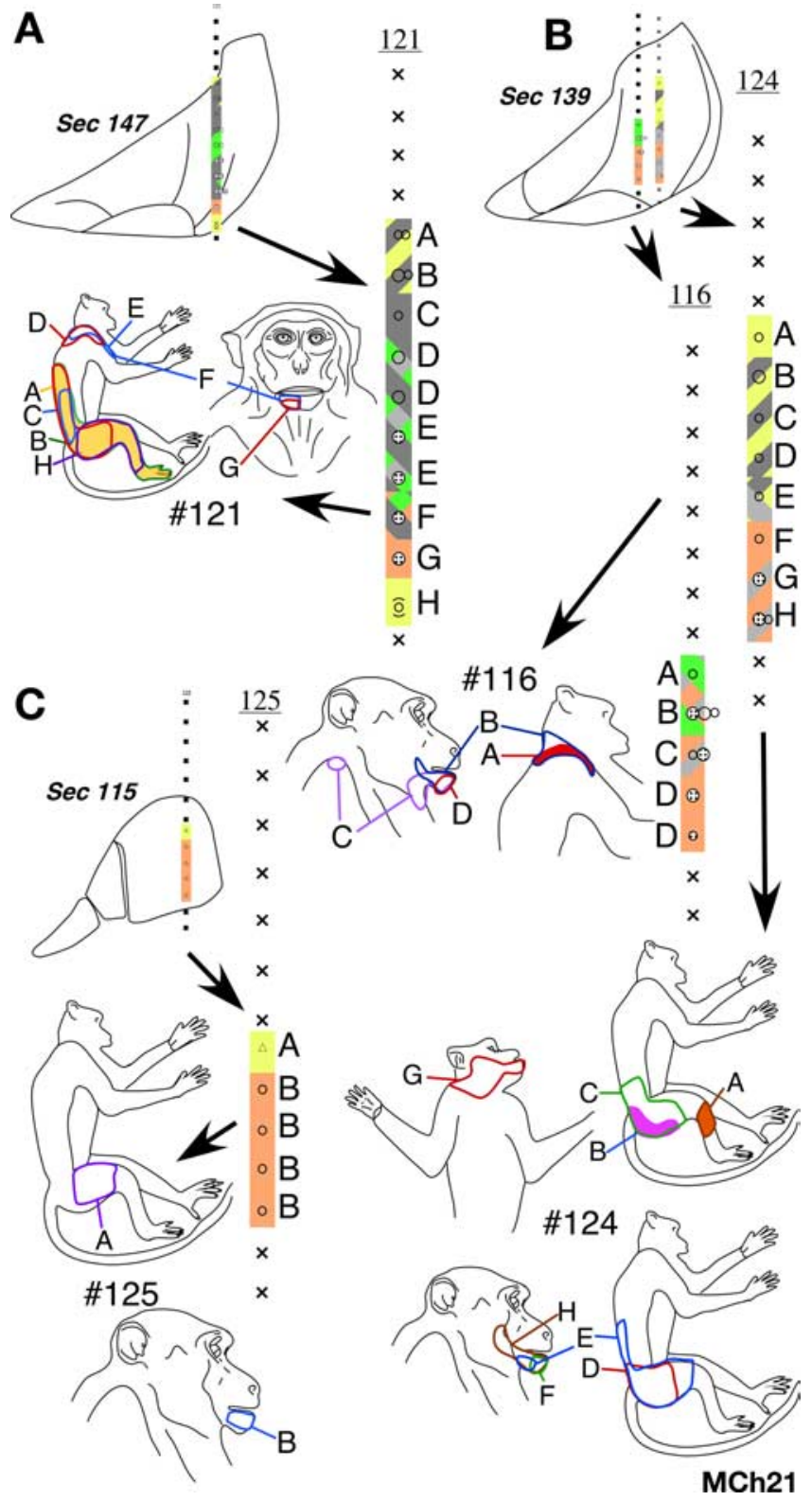

Figure 22. Please see legend to Figure 18.

Flor H, Elbert T, Knecht S, Wienbruch C, Pantev C, Birbaumer N, Larbig W, Taub E (1995) Phantom-limb pain as a perceptual correlate of cortical reorganization following arm amputation. Nature 375:482-484.

Flor H, Braun C, Elbert T, Birbaumer N (1997) Extensive reorganization of primary somatosensory cortex in chronic back pain patients. Neurosci Lett 224:5-8.

Florence SL, Kaas JH (1995) Large-scale reorganization at multiple levels of the somatosensory pathway follows therapeutic amputation of the hand in monkeys. J Neurosci 15:8083-8095.

Florence SL, Wall JT, Kaas JH (1988) The somatotopic pattern of afferent projections from the digits to the spinal cord and cuneate nucleus in macaque monkeys. Brain Res 452:388-392.

Florence SL, Wall JT, Kaas JH (1989) Somatotopic organization of inputs from the hand to the spinal gray and cuneate nucleus of monkeys with observations on the cuneate nucleus of humans. J Comp Neurol 286:48-70.

Florence SL, Taub HB, Kaas JH (1998) Large-scale sprouting of cortical connections after peripheral injury in adult macaque monkeys. Science 282:1117-1121.

Florence SL, Hackett TA, Strata F (2000) Thalamic and cortical contribu-
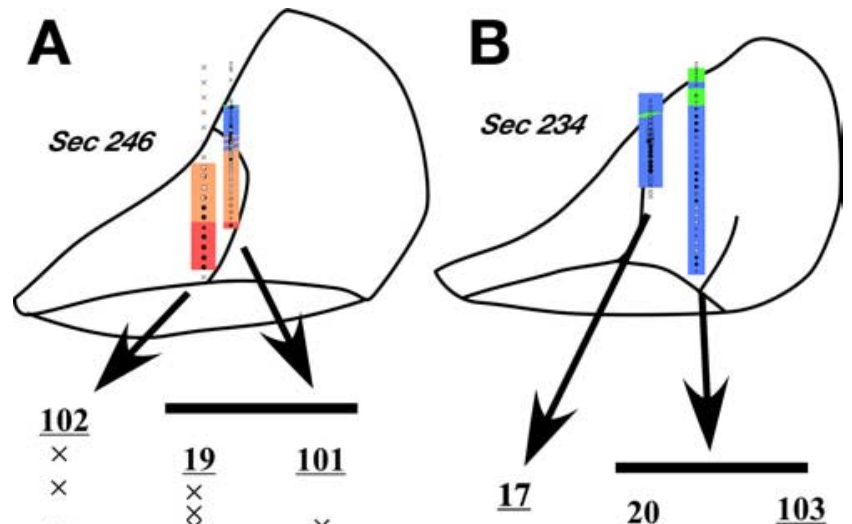

$\times$

$\times \quad \times$

$\times$

$\times$

$$
\times
$$$$
\times
$$$$
x
$$$$
\times
$$$$
x
$$
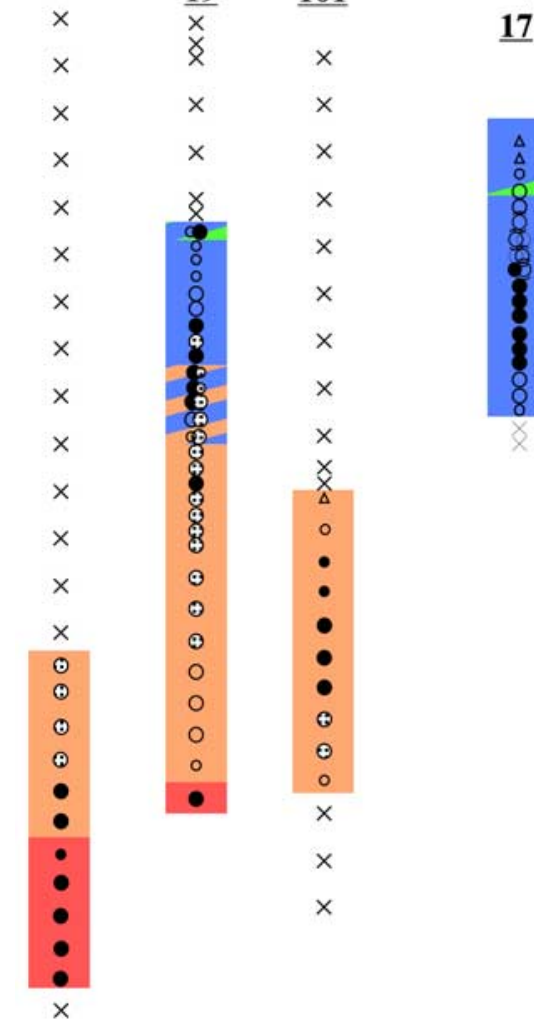

17
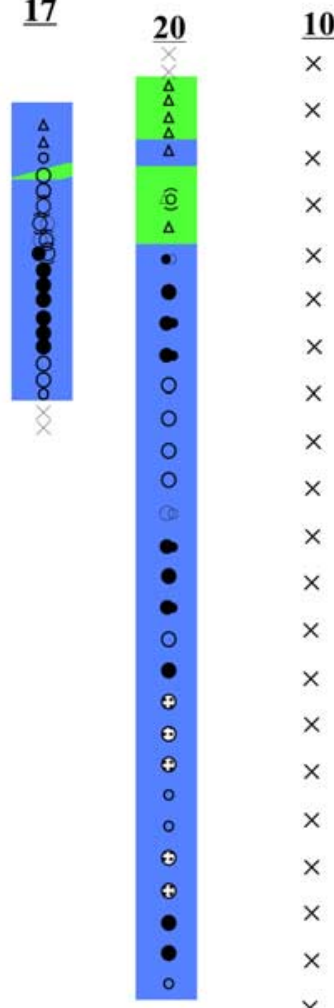

$\underline{103}$

$\times \quad x$

$x \quad x$

$\times$

$\times$

$\times$

$\times$

$\times$

$\times$

$\times$

$\times$

$x$

$\times$

$\times$

$\times$

$\times$

$\times$

$\times$

$\times$

$\times$

$\times$

$\times$

$\times$

$\times$

MAc20 $\times$

Figure 23. $\quad A, B$, Somatotopy in electrode penetrations through the ventroposterior nucleus of monkey MAc20 before and immediately after dorsal column lesion. $A$, Is a more rostral section compared with $\boldsymbol{B}$. Penetration \#19 and \#20 are prelesion penetrations although \#102, \#101, and \#103 were made after lesion. Penetration \#101 was made at the same location as \#19 and penetration \#103 at the location of \#20. Note the absence of receptive fields in the hand (blue) after the lesion. Penetrations within a rostrocaudal extent of $500 \mu \mathrm{m}$ are projected on a single intermediate section. Conventions as for Figures $4 A$ and 17.

tions to neural plasticity after limb amputation. J Neurophysiol 83:3154-3159.

Florence SL, Boydston LA, Hackett TA, Lachoff HT, Strata F, Niblock MM (2001) Sensory enrichment after peripheral nerve injury restores cortical, not thalamic, receptive field organization. Eur J Neurosci 13:1755-1766.

Garraghty PE, Kaas JH (1991a) Functional reorganization in adult monkey thalamus after peripheral nerve injury. Neuroreport 2:747-750.

Garraghty PE, Kaas JH (1991b) Large-scale functional reorganization in adult monkey cortex after peripheral nerve injury. Proc Natl Acad Sci U S A 88:6976-6980.

Garraghty PE, Hanes DP, Florence SL, Kaas JH (1994) Pattern of peripheral 
deafferentation predicts reorganizational limits in adult primate somatosensory cortex. Somatosens Mot Res 11:109-117.

Grüsser SM, Mühlnickel W, Schaefer M, Villringer K, Christmann C, Koeppe C, Flor H (2004) Remote activation of referred phantom sensation and cortical reorganization in human upper extremity amputees. Exp Brain Res 154:97-102.

Jain N (2002) Adult brain plasticity - what is revealed is exciting, what is hidden is critical. J Biosci 27:439-442.

Jain N, Florence SL, Kaas JH (1995) Limits on plasticity in somatosensory cortex of adult rats: hindlimb cortex is not reactivated after dorsal column section. J Neurophysiol 73:1537-1546.

Jain N, Catania KC, Kaas JH (1997) Deactivation and reactivation of somatosensory cortex after dorsal spinal cord injury. Nature 386:495-498.

Jain N, Catania KC, Kaas JH (1998) A histologically visible representation of the fingers and palm in primate area $3 \mathrm{~b}$ and its immutability following long term deafferentation. Cerebral Cortex 8:227-236.

Jain N, Florence SL, Qi HX, Kaas JH (2000) Growth of new brain stem connections in adult monkeys with massive sensory loss. Proc Natl Acad Sci U S A 97:5546-5550.

Jain N, Qi HX, Catania KC, Kaas JH (2001) Anatomical correlates of the face and oral cavity representation in somatosensory area $3 \mathrm{~b}$ of monkeys. J Comp Neurol 429:455-468.

Jones EG (1983) Lack of collateral thalamocortical projections to fields of the first somatic sensory cortex in monkeys. Exp Brain Res 52:375-384.

Jones EG (2000) Cortical and subcortical contributions to activitydependent plasticity in primate somatosensory cortex. Annu Rev Neurosci 23:1-37.

Jones EG, Friedman DP (1982) Projection pattern of functional components of thalamic ventrobasal complex on monkey somatosensory cortex. J Neurophysiol 48:521-544.

Jones EG, Pons TP (1998) Thalamic and brainstem contributions to largescale plasticity of primate somatosensory cortex. Science 282:1121-1125.

Kaas JH, Florence SL, Jain N (1999) Subcortical contributions to massive cortical reorganzations. Neuron 22:657-660.

Kaas JH, Nelson RJ, Sur M, Dykes RW, Merzenich MM (1984) The somatotopic organization of the ventroposterior thalamus of the squirrel monkey, Saimiri sciureus. J Comp Neurol 1984:111-140.

Kaas JH, Qi HX, Burish MJ, Gharbawie OA, Onifer SM, Massey JM (2008) Cortical and subcortical plasticity in the brains of humans, primates, and rats after damage to sensory afferents in the dorsal columns of the spinal cord. Exp Neurol 209:407-416.

Krupa DJ, Ghazanfar AA, Nicolelis MA (1999) Immediate thalamic sensory plasticity depends on corticothalamic feedback. Proc Natl Acad Sci U S A 96:8200-8205.

Lenz FA, Tasker RR, Dostrovsky JO, Kwan HC, Gorecki J, Hirayama T, Murphy JT (1987) Abnormal single-unit activity recorded in the somatosensory thalamus of a quadriplegic patient with central pain. Pain 31:225-236.

Lenz FA, Kwan HC, Martin R, Tasker R, Richardson RT, Dostrovsky JO (1994) Characteristics of somatotopic organization and spontaneous neuronal activity in the region of the thalamic principal sensory nucleus in patients with spinal cord transection. J Neurophysiol 72:1570-1587.

Lenz FA, Garonzik IM, Zirh TA, Dougherty PM (1998) Neuronal activity in the region of the thalamic principal sensory nucleus (ventralis caudalis) in patients with pain following amputations. Neuroscience 86:1065-1081.

Manger PR, Woods TM, Muñoz A, Jones EG (1997) Hand/face border as a limiting boundary in the body representation in monkey somatosensory cortex. J Neurosci 17:6338-6351.

Merzenich MM, Kaas JH, Wall J, Nelson RJ, Sur M, Felleman D (1983a) Topographic reorganization of somatosensory cortical areas $3 \mathrm{~B}$ and 1 in adult monkeys following restricted deafferentation. Neuroscience 8:33-55.

Merzenich MM, Kaas JH, Wall JT, Sur M, Nelson RJ, Felleman DJ (1983b) Progression of change following median nerve section in the cortical representation of the hand in areas $3 \mathrm{~b}$ and 1 in adult owl and squirrel monkeys. Neuroscience 10:639-665.

Merzenich MM, Nelson RJ, Stryker MP, Cynader MS, Schoppmann A, Zook JM (1984) Somatosensory cortical map changes following digit amputation in adult monkeys. J Comp Neurol 224:591-605.

Moore CI, Stern CE, Dunbar C, Kostyk SK, Gehi A, Corkin S (2000) Referred phantom sensations and cortical reorganization after spinal cord injury in humans. Proc Natl Acad Sci U S A 97:14703-14708.

Nelson RJ, Sur M, Felleman DJ, Kaas JH (1980) Representation of the body surface in postcentral parietal cortex of Macaca fascicularis. J Comp Neurol 192:611-643.

Pollin B, Albe-Fessard D (1979) Organization of somatic thalamus in monkeys with and without section of dorsal spinal tracts. Brain Res 173:431-449.

Pons TP, Wall JT, Garraghty PE, Cusick CG, Kaas JH (1987) Consistent features of the representation of the hand in area $3 \mathrm{~b}$ of macaque monkeys. Somatosens Res 4:309-331.

Pons TP, Garraghty PE, Ommaya AK, Kaas JH, Taub E, Mishkin M (1991) Massive cortical reorganization after sensory deafferentation in adult macaques. Science 252:1857-1860.

Qi HX, Kaas JH (2004) Myelin stains reveal an anatomical framework for the representation of the digits in somatosensory area $3 \mathrm{~b}$ of macaque monkeys. J Comp Neurol 477:172-187.

Sherrington E (1939) On the distribution of the sensory nerve-roots. In: Selected writings of Sir Charles Sherrington (D. Denny-Brown, ed), pp 31-93. London: Hamish Hamiston Medical Books.

Silva AC, Rasey SK, Wu X, Wall JT (1996) Initial cortical reactions to injury of the median and radial nerves to the hands of adult primates. J Comp Neurol 366:700-716.

Turnbull BG, Rasmusson DD (1991) Chronic effects of total or partial digit denervation on raccoon somatosensory cortex. Somatosens Mot Res 8:201-213.

Wall JT, Cusick CG (1984) Cutaneous responsiveness in primary somatosensory (S-I) hindpaw cortex before and after partial hindpaw deafferentation in adult rats. J Neurosci 4:1499-1515.

Wall JT, Kaas JH (1985) Cortical reorganization and sensory recovery following nerve damage and regeneration. In: Synaptic plasticity (Cotman CW, ed), pp 231-260. New York: Guilford.

Wall JT, Felleman DJ, Kaas JH (1983) Recovery of normal topography in the somatosensory cortex of monkeys after nerve crush and regeneration. Science 221:771-773.

Wall JT, Kaas JH, Sur M, Nelson RJ, Felleman DJ, Merzenich MM (1986) Functional reorganization in somatosensory cortical areas $3 \mathrm{~b}$ and 1 of adult monkeys after median nerve repair: possible relationships to sensory recovery in humans. J Neurosci 6:218-233.

Weiss T, Miltner WH, Liepert J, Meissner W, Taub E (2004) Rapid functional plasticity in the primary somatomotor cortex and perceptual changes after nerve block. Eur J Neurosci 20:3413-3423.

Weng HR, Lee JI, Lenz FA, Schwartz A, Vierck C, Rowland L, Dougherty PM (2000) Functional plasticity in primate somatosensory thalamus following chronic lesion of the ventral lateral spinal cord. Neuroscience 101:393-401.

Wong-Riley MTT (1979) Changes in the visual system of monocularly sutured or enucleated cats demonstrable with cytochrome oxidase histochemistry. Brain Res 171:11-28. 